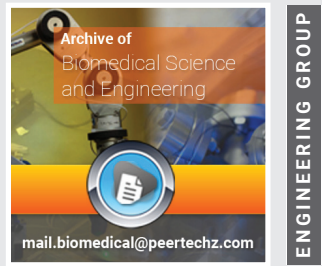

\title{
Some detailed remarks on
}

\section{elemental and core balances}

\section{formulated for non-redox and redox electrolytic systems}

Received: 08 May, 2019

Accepted: 09 May, 2020

Published: 11 May, 2020

*Corresponding author: Tadeusz Michałowski, Department of Analytical Chemistry, Cracow University of Technology, 31-155 Cracow, Poland, E-mail:michalot@o2.pl

Keywords: Electrolytic systems; GATES; GEB; Potentiometric titration

https://www. peertechz.com

\section{Check for updates}

\section{Anna M Michałowska-Kaczmarczyk ${ }^{1}$ and Tadeusz Michałowski2* \\ ${ }^{1}$ Department of Oncology, The University Hospital in Cracow, 31-501 Cracow, Poland \\ ${ }^{2}$ Department of Analytical Chemistry, Cracow University of Technology, 31-155 Cracow, Poland}

\begin{abstract}
Any electrolytic system in aqueous media is described with use of charge balance, $f_{0}=\mathrm{ChB}$, and K elemental or core balances, $f_{\mathrm{k}}=f\left(\mathrm{Y}_{\mathrm{k}}\right)(\mathrm{k}=1, \ldots, \mathrm{K})$. The balances: $f_{1}$ $=f(\mathrm{H})$ and $f_{2}=f(0)$ are the basis to formulate the linear combination $f_{12}=2 \cdot f_{2}-f_{1}=2 \cdot f(0)-f(\mathrm{H})$. For a redox system with $\mathrm{K}-\mathrm{K}^{*}$ electron-active elements (players), $f_{12}$ is linearly independent on $f_{0}, f_{3}, \ldots, f_{K^{\prime}}$. i.e., the redox system is described by $\mathrm{K}$ independent balances $f_{0^{\prime}}, f_{12}, f_{3}, \ldots, f_{K}$. For a non-redox system $\left(K^{*}=K\right), f_{12}$ is linearly dependent on $f_{0}, f_{3}, \ldots, f_{k^{\prime}}$, .e., a redox system is described by $\mathrm{K}-1$ independent balances $f_{0}, f_{3}, \ldots, f_{\mathrm{K}}$. Consequently, the linear combination $f_{12}+f_{0}-\sum_{\mathrm{k}=3}^{\mathrm{k} \cdot} \mathrm{d}_{\mathrm{k}} \cdot f_{\mathrm{k}} \Leftrightarrow \sum_{\mathrm{k}=1}^{\mathrm{k} \cdot} \mathrm{d}_{\mathrm{k}} \cdot f_{\mathrm{k}}-f_{0}$ with $d_{k}$ equal to the oxidation numbers of the related elements, is reducible to identity, $0=0$, for a non-redox system, i.e., $f_{12}$ is not the independent balance in this system. In a redox system, $f_{12}$ is the primary form of generalized electron balance (GEB). To check the linear dependency of the balances $f_{0}, f_{12}, f_{3}, \ldots, f_{k}$, the elemental and core balances are formulated. These regularities are confirmed in a series of electrolytic non-redox and redox systems, of different degree of complexity.
\end{abstract}

\section{Introduction}

The article concerns fundamental regularities, obligatory for electrolytic systems of different complexity, considered from the viewpoint of Generalized Approach to Electrolytic Systems (GATES), formulated by Michałowski (1992) [1,2]. Balancing of an electrolytic system according to GATES, is based on rules of conservation of particular elements, and on a charge balance, expressing the rule of electroneutrality of this system. We refer to aquenus media, $\mathrm{W}=\mathrm{H}_{2} \mathrm{O}$, where the species $\mathrm{X}_{\mathrm{i}}^{\mathrm{z}_{1}}$ exist as hydrates $\mathrm{X}_{\mathrm{i}}^{\mathrm{z}_{1}} \cdot \mathrm{n}_{\mathrm{i} W} ; \mathrm{z}_{\mathrm{i}}=0, \pm 1, \pm 2, \ldots$ is a charge, expressed in elementary charge unit, $\mathrm{e}=\mathrm{F} / \mathrm{N}_{\mathrm{A}}$ ( $\mathrm{F}=$ Faraday's constant, $\mathrm{N}_{\mathrm{A}}=$ Avogadro's number) $; \mathrm{n}_{\mathrm{i}}=\mathrm{n}_{\mathrm{iW}}=\mathrm{n}_{\mathrm{i}} \mathrm{H}_{2} \mathrm{O} \geq \Omega$ is a mean number of water $\left(\mathrm{W}=\mathrm{H}_{2} \mathrm{O}\right)$ molecules attached to $\mathrm{X}_{\mathrm{i}}^{\mathrm{z}_{1}}$. For ordering purposes, we assume $\mathrm{Y}_{1}=\mathrm{H}$ (hydrogen) and $\mathrm{Y}_{2}=\mathrm{O}$ (oxygen), and formulate first the linear combination

$f_{12}=2 \cdot f_{2}-f_{1}=2 \cdot f(\mathrm{O})-f(\mathrm{H})$ of elemental balances: $f_{1}=f(\mathrm{H})$ for hydrogen $(\mathrm{H})$ and $f_{2}=f(\mathrm{O})$ for oxygen (O), related to the system considered. Then the linear combination of $f_{12}$ with charge balance $\left(f_{0}\right)$ and elemental/core balances $f_{\mathrm{k}}=f\left(\mathrm{Y}_{\mathrm{k}}\right)(\mathrm{k}=3, \ldots, \mathrm{K})$ for elements/cores $\mathrm{Y}_{\mathrm{k}} \neq \mathrm{H}$, O will be formulated [3]. A core is considered as a cluster of different atoms with defined composition (expressed by chemical formula), structure and external charge, e.g. $\mathrm{SO}_{4}^{-2}$ is a core with external charge -2 , composed of two elements: $\mathrm{S}$ and $\mathrm{O}$. The balances $f_{\mathrm{k}}(\mathrm{k}=1, \ldots, \mathrm{K})$ interrelate the numbers of different elements/cores in components forming a system, and in the species thus formed. The notation of the balances $\left(f_{0}, f_{1}, \ldots, f_{\mathrm{K}}\right)$ applied here is as follows

$$
\begin{aligned}
& f_{\mathrm{k}}=\left\{\mathrm{s}_{\mathrm{ik}}\right\}-\left\{\mathrm{c}_{\mathrm{jk}}\right\}=0 \Leftrightarrow-f_{\mathrm{k}}=\left\{\mathrm{c}_{\mathrm{jk}}\right\}-\left\{\mathrm{s}_{\mathrm{jk}}\right\}=0 \Leftrightarrow \\
& f_{\mathrm{k}}:\left\{\mathrm{s}_{\mathrm{ik}}\right\}=\left\{\mathrm{c}_{\mathrm{jk}}\right\} \Leftrightarrow-f_{\mathrm{k}}:\left\{\mathrm{c}_{\mathrm{jk}}\right\}=\left\{\mathrm{s}_{\mathrm{ik}}\right\}
\end{aligned}
$$

where $\left\{\mathrm{c}_{\mathrm{jk}}\right\}$ is referred to a set of some components, $\left\{\mathrm{s}_{\mathrm{ik}}\right\}-$ to 
some species in $\mathrm{k}$-th balance; $\mathrm{c}_{\mathrm{io}}=0$, i.e., $\left\{\mathrm{c}_{\mathrm{jk}}\right\}=\phi$ (empty set, for $f_{0}$ ). Numbers of components of $\mathrm{j}$-th kind are denoted by $\mathrm{N}_{\mathrm{oj}}(\mathrm{j}=1, \ldots, \mathrm{J})$, numbers of the species $\mathrm{s}_{\mathrm{ik}}=\mathrm{X}_{\mathrm{i}}^{\mathrm{zi}}$ of $\mathrm{i}$-th kind are denoted by $\mathrm{N}_{\mathrm{i}}(\mathrm{i}=1, \ldots, \mathrm{I})$. For balancing purposes, the hydrated species $\mathrm{X}_{\mathrm{i}}^{\mathrm{zi}} \cdot \mathrm{n}_{\mathrm{iw}}$ in the system are specified as $\mathrm{X}_{\mathrm{i}}^{\mathrm{zi}}\left(\mathrm{N}_{\mathrm{i}}, \mathrm{n}_{\mathrm{i}}\right)$; e.g., the notation $\mathrm{HSO}_{4}^{-1}\left(\mathrm{~N}_{5}, \mathrm{n}_{5}\right)$ applied in Example 1 (below) refers to $\mathrm{N}_{5}$ ions of $\mathrm{HSO}_{4}^{-1} \cdot \mathrm{n}_{5} \mathrm{H}_{2} \mathrm{O}$ involving: $\mathrm{N}_{5}\left(1+2 \mathrm{n}_{5}\right)$ atoms of $\mathrm{H}$, $\mathrm{N}_{5}\left(4+\mathrm{n}_{5}\right)$ atoms of $\mathrm{O}$, and $\mathrm{N}_{5}$ atoms of $\mathrm{S}$. Molar concentration of the species $X_{\mathrm{i}}^{\mathrm{zi}} \cdot \mathrm{n}_{\mathrm{iw}}$ be denoted by $\left[\mathrm{X}_{\mathrm{i}}^{\mathrm{zi}}\right]$.

The $f_{12}$ (Equation 1) is the primary form of Generalized Electron Balance (GEB), $f_{12}=p r-G E B$, formulated (2005) for redox systems according to Approach II to GEB, where prior knowledge of ONs of all elements in components and species is not needed. The Approach II to GEB is fully compatible with the Approach I to GEB [4-15] formulated (1992) and based on the principle of common pool of electrons involved with electronactive elements, named as 'players' and distinguished from electron-non-active elements considered as 'fans', when a redox system is considered according to card game principle [15]; ONs for elements in components and species are needed in the Approach I. The principles of GEB formulation, discovered $(1992,2005)$ by Michałowski and resolved according to GATES as GATES/GEB $[1,16]$, were unknown in earlier literature.

GEB completes the set of $\mathrm{K}$ balances $\left(f_{0}, f_{12}, f_{3}, \ldots, f_{\mathrm{K}}\right)$ as algebraic equations necessary for solving a redox system; $\mathrm{K}-1$ balances $\left(f_{0}, f_{3}, \ldots, f_{\mathrm{K}}\right)$ are required for solving a non-redox system; $f_{12}$ is omitted as derivative (not primary) balance. GEB is the law of Nature related to equilibrium, metastable or kinetic electrolytic redox systems, of any degree of complexity [1,15-24].

In this paper, the general properties of the linear combination of the balances $\left(f_{0}, f_{12}, f_{3}, \ldots, f_{\mathrm{K}}\right)$ related to non-redox and redox systems of different degree of complexity, are discussed. Any redox system is involved with change of oxidation numbers of $\mathrm{K}-\mathrm{K}^{*}$ electron-active elements (players), where $\mathrm{K}^{*}$ is the number of electron-non-active elements (fans). For a nonredox system we have $\mathrm{K}^{*}=\mathrm{K}$, i.e., $\mathrm{K}-\mathrm{K}^{*}=0$. Assuming that $\mathrm{H}$ and $\mathrm{O}$ participate the system as fans, we formulate the linear combination $[15,16,25-33]$

$$
f_{12}+f_{0}-\sum_{\mathrm{k}=3}^{\mathrm{k}^{\cdot}} \mathrm{d}_{\mathrm{k}} \cdot f_{\mathrm{k}}=0 \Leftrightarrow \sum_{\mathrm{k}=1}^{\mathrm{k}} \mathrm{d}_{\mathrm{k}} \cdot f_{\mathrm{k}}-f_{\mathrm{o}}=0
$$

applicable for non-redox and redox systems, of different degree of complexity. For a non-redox system

$$
\sum_{\mathrm{k}=1}^{\mathrm{k}} \mathrm{d}_{\mathrm{k}} \cdot f_{\mathrm{k}}-f_{\mathrm{o}}
$$

is transformed into identity, $0=0$. We prove that the equation (3)

$\left(1^{\circ}\right)$ implies a general criterion distinguishing between non-redox and redox systems;

$\left(2^{\circ}\right)$ defines $d_{k}$ as oxidation numbers (ONs) of particular elements in components and species of a non-redox or redox system.

Synthesis of chemical and physical laws of conservation is expressed, respectively, by the equalities of left and right sides of Equations (3) and (3a).

\section{Formulation of non-redox systems}

Example 1: The (static) system is formed from the following components: $\mathrm{Na}_{2} \mathrm{~S} \cdot 9 \mathrm{H}_{2} \mathrm{O}\left(\mathrm{N}_{01}\right), \mathrm{Na}_{2} \mathrm{SO}_{4} \cdot 10 \mathrm{H}_{2} \mathrm{O}\left(\mathrm{N}_{02}\right), \mathrm{H}_{2} \mathrm{O}\left(\mathrm{N}_{03}\right)$. The species in the system thus formed, namely:

$\mathrm{H}_{2} \mathrm{O}\left(\mathrm{N}_{1}\right), \mathrm{H}^{+1}\left(\mathrm{~N}_{2}, \mathrm{n}_{2}\right), \mathrm{OH}^{-1}\left(\mathrm{~N}_{3}, \mathrm{n}_{3}\right), \mathrm{Na}^{+1}\left(\mathrm{~N}_{4}, \mathrm{n}_{4}\right), \mathrm{HSO}_{4}^{-1}\left(\mathrm{~N}_{5}\right.$, $\left.\mathrm{n}_{5}\right), \mathrm{SO}_{4}^{-2}\left(\mathrm{~N}_{6}, \mathrm{n}_{6}\right), \mathrm{H}_{2} \mathrm{~S}\left(\mathrm{~N}_{7}, \mathrm{n}_{7}\right)$,

$\mathrm{HS}^{-1}\left(\mathrm{~N}_{8}, \mathrm{n}_{8}\right), \mathrm{S}^{-2}\left(\mathrm{~N}_{9}, \mathrm{n}_{9}\right)$

are involved in the following balances:

$f_{0}=\mathrm{ChB}:$

$\mathrm{N}_{2}-\mathrm{N}_{3}+\mathrm{N}_{4}-\mathrm{N}_{5}-2 \mathrm{~N}_{6}-\mathrm{N}_{8}-2 \mathrm{~N}_{9}=0$

$f_{1}=f(\mathrm{H}):$

$2 \mathrm{~N}_{1}+\mathrm{N}_{2}\left(1+2 \mathrm{n}_{2}\right)+\mathrm{N}_{3}\left(1+2 \mathrm{n}_{3}\right)+2 \mathrm{~N}_{4} \mathrm{n}_{4}+\mathrm{N}_{5}\left(1+2 \mathrm{n}_{5}\right)+2 \mathrm{~N}_{6} \mathrm{n}_{6}+$ $\mathrm{N}_{7}\left(2+2 \mathrm{n}_{7}\right)+\mathrm{N}_{8}\left(1+2 \mathrm{n}_{8}\right)+2 \mathrm{~N}_{9} \mathrm{n}_{9}$

$=18 \mathrm{~N}_{01}+20 \mathrm{~N}_{02}+2 \mathrm{~N}_{03}$

$f_{2}=f(0):$

$\mathrm{N}_{1}+\mathrm{N}_{2} \mathrm{n}_{2}+\mathrm{N}_{3}\left(1+\mathrm{n}_{3}\right)+\mathrm{N}_{4} \mathrm{n}_{4}+\mathrm{N}_{5}\left(4+\mathrm{n}_{5}\right)+\mathrm{N}_{6}\left(4+\mathrm{n}_{6}\right)+\mathrm{N}_{7} \mathrm{n}_{7}+$ $\mathrm{N}_{8} \mathrm{n}_{8}+\mathrm{N}_{9} \mathrm{n}_{9}$

$=9 \mathrm{~N}_{01}+14 \mathrm{~N}_{02}+\mathrm{N}_{03}$

$f_{12}=2 \cdot f_{2}-f_{1}:$

$=\mathrm{N}_{2}+\mathrm{N}_{3}+7 \mathrm{~N}_{5}+8 \mathrm{~N}_{6}-2 \mathrm{~N}_{7}-\mathrm{N}_{8}=8 \mathrm{~N}_{02}$

$-f_{3}=-f(\mathrm{Na})$ :

$2 \mathrm{~N}_{01}+2 \mathrm{~N}_{02}=\mathrm{N}_{4}$

$-6 \cdot f_{4}=6 \cdot f\left(\mathrm{SO}_{4}\right):$

$6 \mathrm{~N}_{02}=6 \mathrm{~N}_{5}+6 \mathrm{~N}_{6}$

$2 \cdot f_{5}=2 \cdot f(\mathrm{~S}):$

$2 \mathrm{~N}_{7}+2 \mathrm{~N}_{8}+2 \mathrm{~N}_{9}=2 \mathrm{~N}_{01}$

$f_{12}+f_{0}-f_{3}-6 \cdot f_{4}+2 \cdot f_{5}$

$0=0$

i.e., the linear combination (4) is transformed into identity; it can be rewritten into the form

$$
\begin{aligned}
& \quad(+1) \cdot f_{1}+(-2) \cdot f_{2}+(+1) \cdot f_{3}+(+6) \cdot f_{4}+(-2) \cdot f_{5}-f_{0}=0 \Rightarrow \\
& \quad(+1) \cdot f(\mathrm{H})+(-2) \cdot f(0)+(+1) \cdot f(\mathrm{Na})+(+6) \cdot f\left(\mathrm{SO}_{4}\right)+(-2) \cdot f(\mathrm{~S})- \\
& \mathrm{ChB}=0
\end{aligned}
$$

where the numbers in round brackets as multipliers for $f_{\mathrm{i}}(\mathrm{i}=1, \ldots, 5)$ are equal to ONs of the related elements. Within $f_{12}$, and then within (5), $N_{1}, N_{03}$, water in hydrates and all $n_{i W}$

Citation: AM Michałowska-Kaczmarczyk, Michałowski T (2020) Some detailed remarks on elemental and core balances formulated for non-redox and redox electrolytic systems. Biomed Sci Eng 6(1): 021-033. DOI: https://dx.doi.org/10.17352/abse.000017 
values within components and species, are cancelled. The species $\mathrm{X}_{\mathrm{i}}^{\mathrm{zi}} \cdot \mathrm{n}_{\mathrm{iw}}$ not involving $\mathrm{H}$ and/or $\mathrm{O}$ within $\mathrm{X}_{\mathrm{i}}^{\mathrm{zi}}$, are also cancelled in $f_{12}$. More specifically, the $f_{3}$ involving only one kind of species, is considered here as equality, not equation.

In this system, symproportionation [21] of sulfur does not occur; from this viewpoint, the system is at a metastable state $[1,13]$. Consequently, all elements $(\mathrm{H}, \mathrm{O}, \mathrm{Na}, \mathrm{S})$ involved in this system are perceived as fans, i.e., $\mathrm{K}^{*}=\mathrm{K}=4$. Sulfide oxidation can occur in presence of sulfate-reducing bacteria [34]. Sulfide gives sulfate after oxidation with $\mathrm{H}_{2} \mathrm{O}_{2}$ [35], whereas sulfate ions are not reducible in usual procedure [36].

The elemental balance for S is $f^{*}(S)=f_{45}=f_{4}+f_{5}$. The linear combination

$$
f_{12}+f_{0}-f_{3}+\mathrm{p} \cdot f_{45}
$$

is not transformable into identity $0=0$, at any $\mathrm{p}$-value. From (6) and (4) we get the contradiction: $p=6$ and $p=-2$, i.e., $f_{45}$ is not applicable for checking $f_{12}$ (Eq. 1) as the criterion of independency of the related balances.

Example 2: (dynamic system). $\mathrm{V}_{0} \mathrm{~mL}$ of titrand (D), containing $\mathrm{ZnSO}_{4}\left(\mathrm{C}_{0}\right)+\mathrm{NH}_{3}\left(\mathrm{C}_{1}\right)+\mathrm{NH}_{4} \mathrm{Cl}\left(\mathrm{C}_{2}\right)+\mathrm{NaH}_{2} \mathrm{In}$ (erio $\mathrm{T}$, $\mathrm{C}_{\text {oIn }}$ ) is titrated with $\mathrm{V} \mathrm{mL}$ of titrant (T) containing EDTA (C) [3].

The $\mathrm{D}$ is composed of $\mathrm{N}_{01}$ molecules of $\mathrm{ZnSO}_{4} \cdot 7 \mathrm{H}_{2} \mathrm{O}, \mathrm{N}_{02}$ molecules of $\mathrm{NH}_{3}, \mathrm{~N}_{03}$ molecules of $\mathrm{NH}_{4} \mathrm{Cl}, \mathrm{N}_{04}$ molecules of $\mathrm{NaH}_{2}$ In $=\mathrm{C}_{20} \mathrm{H}_{12} \mathrm{~N}_{3} \mathrm{O}_{7} \mathrm{SNa}, \mathrm{N}_{05}$ molecules of $\mathrm{H}_{2} \mathrm{O}$ and the $\mathrm{T}$ is composed of $\mathrm{N}_{06}$ molecules of EDTA $=\mathrm{Na}_{2} \mathrm{H}_{2} \mathrm{~L} \cdot 2 \mathrm{H}_{2} \mathrm{O}=$ $\mathrm{C}_{10} \mathrm{H}_{14} \mathrm{~N}_{2} \mathrm{O}_{8} \mathrm{Na}_{2} \cdot 2 \mathrm{H}_{2} \mathrm{O}$ and $\mathrm{N}_{07}$ molecules of $\mathrm{H}_{2} \mathrm{O}$, at defined point of titration ( $\mathrm{V} \mathrm{mL}$ of $\mathrm{T}$ added). In the $\mathrm{D}+\mathrm{T}$ system, the following species are formed:

$\mathrm{H}_{2} \mathrm{O}\left(\mathrm{N}_{1}\right), \mathrm{H}^{+1}\left(\mathrm{~N}_{2}, \mathrm{n}_{2}\right), \mathrm{OH}^{-1}\left(\mathrm{~N}_{3}, \mathrm{n}_{3}\right), \mathrm{HSO}_{4}^{-1}\left(\mathrm{~N}_{4}, \mathrm{n}_{4}\right), \mathrm{SO}_{4}$ ${ }^{2}\left(\mathrm{~N}_{5}, \mathrm{n}_{5}\right), \mathrm{Cl}^{-1}\left(\mathrm{~N}_{6}, \mathrm{n}_{6}\right), \mathrm{Na}^{+1}\left(\mathrm{~N}_{7}, \mathrm{n}_{7}\right), \mathrm{NH}_{4}^{+1}\left(\mathrm{n}_{8}, \mathrm{~N}_{8}\right), \mathrm{NH}_{3}\left(\mathrm{n}_{9}\right.$, $\left.\mathrm{N}_{9}\right), \mathrm{Zn}^{+2}\left(\mathrm{~N}_{10}, \mathrm{n}_{10}\right), \mathrm{ZnOH}^{+1}\left(\mathrm{~N}_{11}, \mathrm{n}_{11}\right)$, soluble complex $\mathrm{Zn}(\mathrm{OH})_{2}$ $\left(\mathrm{N}_{12}, \mathrm{n}_{12}\right), \mathrm{Zn}(\mathrm{OH})_{3}^{-1}\left(\mathrm{~N}_{13}, \mathrm{n}_{13}\right), \mathrm{Zn}(\mathrm{OH})_{4}^{-2}\left(\mathrm{~N}_{14}, \mathrm{n}_{14}\right), \mathrm{ZnNH}_{3}{ }^{+2}\left(\mathrm{~N}_{15}\right.$, $\left.\mathrm{n}_{15}\right), \mathrm{Zn}\left(\mathrm{NH}_{3}\right)_{2}{ }^{+2}\left(\mathrm{~N}_{16}, \mathrm{n}_{16}\right), \mathrm{Zn}\left(\mathrm{NH}_{3}\right)_{3}{ }^{+2}\left(\mathrm{~N}_{17}, \mathrm{n}_{17}\right), \mathrm{Zn}\left(\mathrm{NH}_{3}\right)_{4}^{+2}\left(\mathrm{~N}_{18}\right.$, $\left.\mathrm{n}_{18}\right), \mathrm{ZnCl}^{+1}\left(\mathrm{~N}_{19}, \mathrm{n}_{19}\right) ; \mathrm{ZnSO}_{4}\left(\mathrm{~N}_{20}, \mathrm{n}_{20}\right), \mathrm{C}_{20} \mathrm{H}_{13} \mathrm{~N}_{3} \mathrm{O}_{7} \mathrm{~S}\left(\mathrm{~N}_{21}, \mathrm{n}_{21}\right)$, $\mathrm{C}_{20} \mathrm{H}_{12} \mathrm{~N}_{3} \mathrm{O}_{7} \mathrm{~S}^{-1}\left(\mathrm{~N}_{22}, \mathrm{n}_{22}\right), \mathrm{C}_{20} \mathrm{H}_{11} \mathrm{~N}_{3} \mathrm{O}_{7} \mathrm{~S}^{-2}\left(\mathrm{~N}_{23}, \mathrm{n}_{23}\right), \mathrm{C}_{20} \mathrm{H}_{10} \mathrm{~N}_{3} \mathrm{O}_{7} \mathrm{~S}^{-3}$ $\left(\mathrm{N}_{24}, \mathrm{n}_{24}\right), \mathrm{C}_{20} \mathrm{H}_{10} \mathrm{~N}_{3} \mathrm{O}_{7} \mathrm{SZn}^{-1}\left(\mathrm{~N}_{25}, \mathrm{n}_{25}\right),\left(\mathrm{C}_{20} \mathrm{H}_{10} \mathrm{~N}_{3} \mathrm{O}_{7} \mathrm{~S}\right)_{2} \mathrm{Zn}^{-4}\left(\mathrm{~N}_{26}\right.$, $\left.\mathrm{n}_{26}\right), \mathrm{C}_{10} \mathrm{H}_{18} \mathrm{~N}_{2} \mathrm{O}_{8}^{+2}\left(\mathrm{H}_{6} \mathrm{~L}^{+2}\right)$

$\left(\mathrm{N}_{27}, \mathrm{n}_{27}\right), \mathrm{C}_{10} \mathrm{H}_{17} \mathrm{~N}_{2} \mathrm{O}_{8}^{+1}\left(\mathrm{H}_{5} \mathrm{~L}^{+1}\right)\left(\mathrm{N}_{28}, \mathrm{n}_{28}\right), \mathrm{C}_{10} \mathrm{H}_{16} \mathrm{~N}_{2} \mathrm{O}_{8}\left(\mathrm{H}_{4} \mathrm{~L}\right)$ $\left(\mathrm{N}_{29}, \mathrm{n}_{29}\right), \mathrm{C}_{10} \mathrm{H}_{15} \mathrm{~N}_{2} \mathrm{O}_{8}^{-1}\left(\mathrm{H}_{3} \mathrm{~L}^{-1}\right)\left(\mathrm{N}_{30}, \mathrm{n}_{30}\right), \mathrm{C}_{10} \mathrm{H}_{14} \mathrm{~N}_{2} \mathrm{O}_{8}^{-2}\left(\mathrm{H}_{2} \mathrm{~L}^{-2}\right)\left(\mathrm{N}_{31}\right.$ $\left.\mathrm{n}_{31}\right), \mathrm{C}_{10} \mathrm{H}_{13} \mathrm{~N}_{2} \mathrm{O}_{8}^{-3}\left(\mathrm{HL}^{-3}\right)\left(\mathrm{N}_{32}, \mathrm{n}_{32}\right), \mathrm{C}_{10} \mathrm{H}_{12} \mathrm{~N}_{2} \mathrm{O}_{8}^{-4}\left(\mathrm{~L}^{-4}\right)\left(\mathrm{N}_{33}, \mathrm{n}_{33}\right)$, $\mathrm{C}_{10} \mathrm{H}_{13} \mathrm{~N}_{2} \mathrm{O}_{8} \mathrm{Zn}^{-1}\left(\mathrm{ZnHL}^{-1}\right)\left(\mathrm{N}_{34}, \mathrm{n}_{34}\right), \mathrm{C}_{10} \mathrm{H}_{12} \mathrm{~N}_{2} \mathrm{O}_{8} \mathrm{Zn}^{-2}\left(\mathrm{ZnL}^{-2}\right)\left(\mathrm{N}_{35}\right.$, $\left.\mathrm{n}_{35}\right), \mathrm{C}_{10} \mathrm{H}_{13} \mathrm{~N}_{2} \mathrm{O}_{9} \mathrm{Zn}^{-3}\left(\mathrm{ZnOHL}^{-3}\right)\left(\mathrm{N}_{36}, \mathrm{n}_{36}\right)$.

In particular, the complex $\mathrm{ZnOHL}^{-3}$ is formed from $\mathrm{ZnOH}^{+1}$ and $\mathrm{L}^{-4}$.

The charge (ChB), elemental $(f(\mathrm{H}), f(\mathrm{O}), f(\mathrm{Zn}), f(\mathrm{Cl}), f(\mathrm{Na}))$ and core $\left(f\left(\mathrm{SO}_{4}\right), f\left(\mathrm{NH}_{3}\right), f\left(\mathrm{C}_{20} \mathrm{H}_{10} \mathrm{~N}_{3} \mathrm{O}_{7} \mathrm{~S}\right), f\left(\mathrm{C}_{10} \mathrm{H}_{12} \mathrm{~N}_{2} \mathrm{O}_{8}\right)\right)$ balances are written here as follows:

$f_{0}=\mathrm{ChB}:$

$\mathrm{N}_{2}-\mathrm{N}_{3}-\mathrm{N}_{4}-2 \mathrm{~N}_{5}-\mathrm{N}_{6}+\mathrm{N}_{7}+\mathrm{N}_{8}+2 \mathrm{~N}_{10}+\mathrm{N}_{11}-\mathrm{N}_{13}-2 \mathrm{~N}_{14}+$ $2 \mathrm{~N}_{15}+2 \mathrm{~N}_{16}+2 \mathrm{~N}_{17}+2 \mathrm{~N}_{18}+\mathrm{N}_{19}-\mathrm{N}_{22}-2 \mathrm{~N}_{23}$
$-3 \mathrm{~N}_{24}-\mathrm{N}_{25}-4 \mathrm{~N}_{26}+2 \mathrm{~N}_{27}+\mathrm{N}_{28}-\mathrm{N}_{30}-2 \mathrm{~N}_{31}-3 \mathrm{~N}_{32}-4 \mathrm{~N}_{33}$ $-\mathrm{N}_{34}-2 \mathrm{~N}_{35}-3 \mathrm{~N}_{36}=0$

$f_{1}=f(\mathrm{H})$ :

$2 \mathrm{~N}_{1}+\mathrm{N}_{2}\left(1+2 \mathrm{n}_{2}\right)+\mathrm{N}_{3}\left(1+2 \mathrm{n}_{3}\right)+\mathrm{N}_{4}\left(1+2 \mathrm{n}_{4}\right)+2 \mathrm{~N}_{5} \mathrm{n}_{5}+2 \mathrm{~N}_{6} \mathrm{n}_{6}$ $+2 \mathrm{~N}_{7} \mathrm{n}_{7}+\mathrm{N}_{8}\left(4+2 \mathrm{n}_{8}\right)+\mathrm{N}_{9}\left(3+2 \mathrm{n}_{9}\right)+2 \mathrm{~N}_{10} \mathrm{n}_{10}$

$+\mathrm{N}_{11}\left(1+2 \mathrm{n}_{11}\right)+\mathrm{N}_{12}\left(2+2 \mathrm{n}_{12}\right)+\mathrm{N}_{13}\left(3+2 \mathrm{n}_{13}\right)+\mathrm{N}_{14}\left(4+2 \mathrm{n}_{14}\right)+$ $\mathrm{N}_{15}\left(3+2 \mathrm{n}_{15}\right)+\mathrm{N}_{16}\left(6+2 \mathrm{n}_{16}\right)+\mathrm{N}_{17}\left(9+2 \mathrm{n}_{17}\right)$

$+\mathrm{N}_{18}\left(12+2 \mathrm{n}_{18}\right)+2 \mathrm{~N}_{19} \mathrm{n}_{19}+2 \mathrm{~N}_{20} \mathrm{n}_{20}+\mathrm{N}_{21}\left(13+2 \mathrm{n}_{21}\right)+\mathrm{N}_{22}(12+$ $\left.2 n_{22}\right)+N_{23}\left(11+2 n_{23}\right)+N_{24}\left(10+2 n_{24}\right)$

$+\mathrm{N}_{25}\left(10+2 \mathrm{n}_{25}\right)+\mathrm{N}_{26}\left(20+2 \mathrm{n}_{26}\right)+\mathrm{N}_{27}\left(18+2 \mathrm{n}_{27}\right)+\mathrm{N}_{28}(17+$ $\left.2 \mathrm{n}_{28}\right)+\mathrm{N}_{29}\left(16+2 \mathrm{n}_{29}\right)+\mathrm{N}_{30}\left(15+2 \mathrm{n}_{30}\right)$

$+\mathrm{N}_{31}\left(14+2 \mathrm{n}_{31}\right)+\mathrm{N}_{32}\left(13+2 \mathrm{n}_{32}\right)+\mathrm{N}_{33}\left(12+2 \mathrm{n}_{33}\right)+\mathrm{N}_{34}(13+$ $\left.2 \mathrm{n}_{34}\right)+\mathrm{N}_{35}\left(12+2 \mathrm{n}_{35}\right)+\mathrm{N}_{36}\left(13+2 \mathrm{n}_{36}\right)$

$=14 \mathrm{~N}_{01}+3 \mathrm{~N}_{02}+4 \mathrm{~N}_{03}+12 \mathrm{~N}_{04}+2 \mathrm{~N}_{05}+18 \mathrm{~N}_{06}+2 \mathrm{~N}_{07}$

$f_{2}=f(0):$

$\mathrm{N}_{1}+\mathrm{N}_{2} \mathrm{n}_{2}+\mathrm{N}_{3}\left(1+\mathrm{n}_{3}\right)+\mathrm{N}_{4}\left(4+\mathrm{n}_{4}\right)+\mathrm{N}_{5}\left(4+\mathrm{n}_{5}\right)+\mathrm{N}_{6} \mathrm{n}_{6}+\mathrm{N}_{7} \mathrm{n}_{7}$ $+\mathrm{N}_{8} \mathrm{n}_{8}+\mathrm{N}_{9} \mathrm{n}_{9}+\mathrm{N}_{10} \mathrm{n}_{10}$

$+\mathrm{N}_{11}\left(1+\mathrm{n}_{11}\right)+\mathrm{N}_{12}\left(2+\mathrm{n}_{12}\right)+\mathrm{N}_{13}\left(3+\mathrm{n}_{13}\right)+\mathrm{N}_{14}\left(4+\mathrm{n}_{14}\right)+\mathrm{N}_{15} \mathrm{n}_{15}$ $+\mathrm{N}_{16} \mathrm{n}_{16}+\mathrm{N}_{17} \mathrm{n}_{17}$

$+\mathrm{N}_{18} \mathrm{n}_{18}+\mathrm{N}_{19} \mathrm{n}_{19}+\mathrm{N}_{20}\left(4+\mathrm{n}_{20}\right)+\mathrm{N}_{21}\left(7+\mathrm{n}_{21}\right)+\mathrm{N}_{22}\left(7+\mathrm{n}_{22}\right)+$ $\mathrm{N}_{23}\left(7+\mathrm{n}_{23}\right)+\mathrm{N}_{24}\left(7+\mathrm{n}_{24}\right)$

$+\mathrm{N}_{25}\left(7+\mathrm{n}_{25}\right)+\mathrm{N}_{26}\left(14+\mathrm{n}_{26}\right)+\mathrm{N}_{27}\left(8+\mathrm{n}_{27}\right)+\mathrm{N}_{28}\left(8+\mathrm{n}_{28}\right)+$ $\mathrm{N}_{29}\left(8+\mathrm{n}_{29}\right)+\mathrm{N}_{30}\left(8+\mathrm{n}_{30}\right)$

$+\mathrm{N}_{31}\left(8+\mathrm{n}_{31}\right)+\mathrm{N}_{32}\left(8+\mathrm{n}_{32}\right)+\mathrm{N}_{33}\left(8+\mathrm{n}_{33}\right)+\mathrm{N}_{34}\left(8+\mathrm{n}_{34}\right)+$ $\mathrm{N}_{35}\left(8+\mathrm{n}_{35}\right)+\mathrm{N}_{36}\left(9+\mathrm{n}_{36}\right)$

$=11 \mathrm{~N}_{01}+7 \mathrm{~N}_{04}+\mathrm{N}_{05}+10 \mathrm{~N}_{06}+\mathrm{N}_{07}$

$-2 \cdot f_{3}=-2 \cdot f(\mathrm{Zn})$

$2 \mathrm{~N}_{01}=2 \mathrm{~N}_{10}+2 \mathrm{~N}_{11}+2 \mathrm{~N}_{12}+2 \mathrm{~N}_{13}+2 \mathrm{~N}_{14}+2 \mathrm{~N}_{15}+2 \mathrm{~N}_{16}+2 \mathrm{~N}_{17}+$ $2 \mathrm{~N}_{18}+2 \mathrm{~N}_{19}+2 \mathrm{~N}_{20}+2 \mathrm{~N}_{25}+2 \mathrm{~N}_{26}$

$+2 \mathrm{~N}_{34}+2 \mathrm{~N}_{35}+2 \mathrm{~N}_{36}$

$f_{4}=f(\mathrm{Cl})$ :

$\mathrm{N}_{6}+\mathrm{N}_{19}=\mathrm{N}_{03}$

$-f_{5}=-f(\mathrm{Na}):$

$\mathrm{N}_{04}+2 \mathrm{~N}_{06}=\mathrm{N}_{7}$

$-6 \cdot f_{6}=-6 \cdot f\left(\mathrm{SO}_{4}\right):$

$6 \mathrm{~N}_{01}=6 \mathrm{~N}_{4}+6 \mathrm{~N}_{5}+6 \mathrm{~N}_{20}$

$3 \cdot f_{7}=3 \cdot f\left(\mathrm{NH}_{3}\right):$

$3 \mathrm{~N}_{8}+3 \mathrm{~N}_{9}+3 \mathrm{~N}_{15}+6 \mathrm{~N}_{16}+9 \mathrm{~N}_{17}+12 \mathrm{~N}_{18}=3 \mathrm{~N}_{02}+3 \mathrm{~N}_{03}$

$-f_{8}=-f\left(\mathrm{C}_{20} \mathrm{H}_{10} \mathrm{~N}_{3} \mathrm{O}_{7} \mathrm{~S}\right)$ : 


$$
\begin{aligned}
& \mathrm{N}_{04}=\mathrm{N}_{21}+\mathrm{N}_{22}+\mathrm{N}_{23}+\mathrm{N}_{24}+\mathrm{N}_{25}+2 \mathrm{~N}_{26} \\
& 0 \cdot f_{9}=0 \cdot f\left(\mathrm{C}_{10} \mathrm{H}_{12} \mathrm{~N}_{2} \mathrm{O}_{8}\right): \\
& 0 \cdot \mathrm{N}_{27}+0 \cdot \mathrm{N}_{28}+0 \cdot \mathrm{N}_{29}+0 \cdot \mathrm{N}_{30}+0 \cdot \mathrm{N}_{31}+0 \cdot \mathrm{N}_{32}+0 \cdot \mathrm{N}_{33}+0 \cdot \mathrm{N}_{34}+ \\
& 0 \cdot \mathrm{N}_{35}+0 \cdot \mathrm{N}_{36}=0 \cdot \mathrm{N}_{06}
\end{aligned}
$$

\section{The balance}

$$
f_{12}=2 \cdot f(\mathrm{O})-f(\mathrm{H}):
$$

$-\mathrm{N}_{2}+\mathrm{N}_{3}+7 \mathrm{~N}_{4}+8 \mathrm{~N}_{5}-4 \mathrm{~N}_{8}-3 \mathrm{~N}_{9}+\mathrm{N}_{11}+2 \mathrm{~N}_{12}+3 \mathrm{~N}_{13}+4 \mathrm{~N}_{14}$ $-3 \mathrm{~N}_{15}-6 \mathrm{~N}_{16}-9 \mathrm{~N}_{17}-12 \mathrm{~N}_{18}+8 \mathrm{~N}_{20}+\mathrm{N}_{21}$

$+2 \mathrm{~N}_{22}+3 \mathrm{~N}_{23}+4 \mathrm{~N}_{24}+4 \mathrm{~N}_{25}+8 \mathrm{~N}_{26}-2 \mathrm{~N}_{27}-\mathrm{N}_{28}+\mathrm{N}_{30}+2 \mathrm{~N}_{31}+$ $3 \mathrm{~N}_{32}+4 \mathrm{~N}_{33}+3 \mathrm{~N}_{34}+4 \mathrm{~N}_{35}+5 \mathrm{~N}_{36}$

$$
\begin{aligned}
& =8 \mathrm{~N}_{01}-3 \mathrm{~N}_{02}-4 \mathrm{~N}_{03}+2 \mathrm{~N}_{04}+2 \mathrm{~N}_{06} \\
& f_{0}+f_{12}-2 \cdot f_{3}+f_{4}-f_{5}-6 \cdot f_{6}+3 \cdot f_{7}-f_{8}+0 \cdot f_{9}
\end{aligned}
$$

$0=0$

i.e., the the linear combination (10) is transformed into identity, $0=0$. It can be rewritten into the form

$$
(+1) \cdot f_{1}+(-2) \cdot f_{2}+(+2) \cdot f_{3}+(-1) \cdot f_{4}+(+1) \cdot f_{5}+(+6) \cdot f_{6}+(-3) \cdot f_{7}+
$$
$(+1) \cdot f_{8}+(0) \cdot f_{9}-f_{0}=0$

i.e. the numbers in round brackets as multipliers for $f_{\mathrm{i}}$ $(\mathrm{i}=1, \ldots, 9)$ are equal to ONs of the elements or charges ascribed to $\mathrm{S}$ in $\mathrm{SO}_{4}^{-2}, \mathrm{~N}$ in $\mathrm{NH}_{3}, \mathrm{C}_{20} \mathrm{~N}_{3} \mathrm{~S}$ in all the species of erio $\mathrm{T}, \mathrm{C}_{10} \mathrm{~N}_{2}$ in all the species of EDTA.

This system needs some further comments. All elements $(\mathrm{H}, \mathrm{O}, \mathrm{Na}, \mathrm{Cl}, \mathrm{Zn}, \mathrm{S}, \mathrm{N}, \mathrm{C})$ in involved in the components and species the system are considered as fans, i.e., $\mathrm{K}^{*}=\mathrm{K}=8$. The number, 8 , of the elements is lower than the total number of charge and elemental/core balances $(K=1+9=10)$.

The formula $\mathrm{C}_{20} \mathrm{H}_{13} \mathrm{~N}_{3} \mathrm{O}_{7} \mathrm{~S}$ for the neutral species of erio $\mathrm{T}$ can be rewritten into the form $\left(\mathrm{C}_{20} \mathrm{~N}_{3} \mathrm{~S}\right)\left(\mathrm{H}_{13} \mathrm{O}_{7}\right) \Leftrightarrow\left(\mathrm{C}_{20} \mathrm{~N}_{3} \mathrm{~S}\right)_{1}\left(\mathrm{H}_{2} \mathrm{O}\right)_{6} \mathrm{OH}$. The group of elements within $\mathrm{C}_{20} \mathrm{~N}_{3} \mathrm{~S}$ has the net charge $\mathrm{x}$ calculated from the equation $1 \cdot \mathrm{x}+6 \cdot(0)+1 \cdot(-2+1)=0 \Rightarrow \mathrm{x}=+1$. Similarly, in $\mathrm{C}_{10} \mathrm{H}_{16} \mathrm{~N}_{2} \mathrm{O}_{8}=\left(\mathrm{C}_{10} \mathrm{~N}_{2}\right)\left(\mathrm{H}_{16} \mathrm{O}_{8}\right)=\left(\mathrm{C}_{10} \mathrm{~N}_{2}\right)_{1}\left(\mathrm{H}_{2} \mathrm{O}\right)_{8}$, the net charge $\mathrm{x}$ of the $\mathrm{C}_{10} \mathrm{~N}_{2}$ group is calculated from the equation $1 \cdot \mathrm{x}+$ $8 \cdot 0=0$, i.e., $\mathrm{x}=0$. The $\mathrm{C}_{20} \mathrm{~N}_{3} \mathrm{~S}^{+1}$ and $\mathrm{C}_{10} \mathrm{~N}_{2}$ can be also (optionally) considered as cores.

The $f_{6}, f_{7}, f_{8}, f_{9}$ are specified separately, for different cores: $\mathrm{SO}_{4}^{-2}, \mathrm{NH}_{3}, \mathrm{C}_{20} \mathrm{H}_{10} \mathrm{~N}_{3} \mathrm{O}_{7} \mathrm{~S}^{-3}, \mathrm{C}_{10} \mathrm{H}_{12} \mathrm{~N}_{2} \mathrm{O}_{8}^{-4}$, resp. Note that $\mathrm{S}$ enters the compounds and species in $f_{6}, f_{8} ; \mathrm{N}$ enters the compounds and species in $\mathrm{f}_{7}, f_{8}, f_{9}$; $\mathrm{C}$ enters the compounds and species in $f_{8}, f_{9}$. Furthermore, none transformations occur between the cores of the species belonging to separate concentration balances.

Referring again to the species involved with erio $\mathrm{T}$, one can write the elemental balances: $\mathrm{N}_{21}+\mathrm{N}_{22}+\mathrm{N}_{23}+\mathrm{N}_{24}+\mathrm{N}_{25}+2 \mathrm{~N}_{26}=$ $\mathrm{N}_{04}$ (for S); $3 \mathrm{~N}_{21}+3 \mathrm{~N}_{22}+3 \mathrm{~N}_{23}+3 \mathrm{~N}_{24}+3 \mathrm{~N}_{25}+6 \mathrm{~N}_{26}=3 \mathrm{~N}_{04}$ (for $\mathrm{N}$ ); $20 \mathrm{~N}_{21}+20 \mathrm{~N}_{22}+20 \mathrm{~N}_{23}+20 \mathrm{~N}_{24}+20 \mathrm{~N}_{25}+40 \mathrm{~N}_{26}=20 \mathrm{~N}_{04}$ (for C). All the equations are identical and equivalent to Eq. (8), because the core $\mathrm{C}_{20} \mathrm{H}_{10} \mathrm{~N}_{3} \mathrm{O}_{7} \mathrm{~S}$ is unchanged in reactions occurred during the titration. Similarly, the species involved with EDTA fulfill the relations: $10 \mathrm{~N}_{27}+10 \mathrm{~N}_{28}+10 \mathrm{~N}_{29}+10 \mathrm{~N}_{30}+10 \mathrm{~N}_{31}+10 \mathrm{~N}_{32}+$ $10 \mathrm{~N}_{33}+10 \mathrm{~N}_{34}+10 \mathrm{~N}_{35}+10 \mathrm{~N}_{36}=10 \mathrm{~N}_{06}$ (for $\mathrm{C}$ ), and $3 \mathrm{~N}_{27}+3 \mathrm{~N}_{28}+$ $3 \mathrm{~N}_{29}+3 \mathrm{~N}_{30}+3 \mathrm{~N}_{31}+3 \mathrm{~N}_{32}+3 \mathrm{~N}_{33}+3 \mathrm{~N}_{34}+3 \mathrm{~N}_{35}+3 \mathrm{~N}_{36}=3 \mathrm{~N}_{06}$ (for $\mathrm{N})$. Both equations are equivalent to $f_{9}$, Eq. (9).

Denoting the elemental balances for $\mathrm{S}, \mathrm{N}$ and $\mathrm{C}$ as $f^{*}(\mathrm{~S})=$ $f_{68}, f(\mathrm{~N})=f_{789}, f(\mathrm{C})=f_{89}$, we have the relations, expressed in the matrix form as follows

$$
\left[\begin{array}{l}
f_{68} \\
f_{789} \\
f_{89}
\end{array}\right]=\left[\begin{array}{cccc}
1 & 0 & 1 & 0 \\
0 & 1 & 3 & 2 \\
0 & 0 & 20 & 10
\end{array}\right]=\left[\begin{array}{l}
f_{6} \\
f_{7} \\
f_{8} \\
f_{9}
\end{array}\right]
$$

One can prove that the linear combination

$$
f_{0}+f_{12}-2 \cdot f_{3}+f_{4}-f_{5}+\mathrm{p} \cdot f_{68}+\mathrm{q} \cdot f_{789}+\mathrm{r} \cdot f_{89}
$$

is not transformable into identity $0=0$, at any of the $(\mathrm{p}, \mathrm{q}, \mathrm{r}$ ) values. Namely, from Equations (10) and (12) we get

$$
\mathrm{p}\left(f_{6}+f_{8}\right)+\mathrm{q}\left(f_{7}+3 f_{8}+2 f_{9}\right)+\mathrm{r}\left(20 f_{8}+10 f_{9}\right)=\mathrm{p} \cdot f_{6}+\mathrm{q} \cdot f_{7}+
$$
$(\mathrm{p}+3 \mathrm{q}+20 \mathrm{r}) \cdot f_{8}+(2 \mathrm{q}+10 \mathrm{r}) \cdot f_{9}$

$-6 \cdot f_{6}+3 \cdot f_{7}-f_{8}+0 \cdot f_{9} \Rightarrow \mathrm{p}=-6, \mathrm{q}=3$, and $\mathrm{p}+3 \mathrm{q}+20 \mathrm{r}=-1$, $2 \mathrm{q}+10 \mathrm{r}=0$

$r=(-1-(-6)-3 \cdot 3) / 20=-0.2 ; r=-2 \cdot 3 / 10=-0.6$.

The $r=-0.2$ and $r=-0.6$ are contradictory values. Then the option given by Eq. 12, with $f_{68}, f_{789}, f_{89}$, is not applicable for checking $f_{12}$ (Eq. 1) as the criterion of independency of the related balances.

\section{Formulation of redox systems}

Example 3: We consider here

$\left(1^{\circ}\right)$ T subsystem (volume $\mathrm{V} \mathrm{mL}$ ), composed of $\mathrm{KMnO}_{4}\left(\mathrm{~N}_{01}\right)$ $+\mathrm{H}_{2} \mathrm{O}\left(\mathrm{N}_{02}\right)+\mathrm{CO}_{2}\left(\mathrm{~N}_{03}\right)$;

$\left(2^{\circ}\right)$ D subsystem $\left(\mathrm{V}_{0} \mathrm{~mL}\right)$, composed of $\mathrm{FeSO}_{4} \cdot 7 \mathrm{H}_{2} \mathrm{O}\left(\mathrm{N}_{04}\right)+$ $\mathrm{H}_{2} \mathrm{SO}_{4}\left(\mathrm{~N}_{05}\right)+\mathrm{H}_{2} \mathrm{O}\left(\mathrm{N}_{06}\right)+\mathrm{CO}_{2}\left(\mathrm{~N}_{07}\right)$;

and

$\left(3^{\circ}\right) \mathrm{D}+\mathrm{T}$ redox system $\left(\mathrm{V}_{0}+\mathrm{V} \mathrm{mL}\right)$, as the mixture of $\mathrm{D}$ and $\mathrm{T}$, where the following species are formed:

$\mathrm{H}_{2} \mathrm{O}\left(\mathrm{N}_{1}\right) ; \mathrm{H}^{+1}\left(\mathrm{~N}_{2}, \mathrm{n}_{2}\right), \mathrm{OH}^{-1}\left(\mathrm{~N}_{3}, \mathrm{n}_{3}\right), \mathrm{HSO}_{4}^{-1}\left(\mathrm{~N}_{4}, \mathrm{n}_{4}\right), \mathrm{SO}_{4}^{-2}\left(\mathrm{~N}_{5}\right.$, $\left.\mathrm{n}_{5}\right), \mathrm{H}_{2} \mathrm{CO}_{3}\left(\mathrm{~N}_{6}, \mathrm{n}_{6}\right), \mathrm{HCO}_{3}^{-1}\left(\mathrm{~N}_{7}, \mathrm{n}_{7}\right)$,

$\mathrm{CO}_{3}^{-2}\left(\mathrm{~N}_{8}, \mathrm{n}_{8}\right), \mathrm{Fe}^{+2}\left(\mathrm{~N}_{9}, \mathrm{n}_{9}\right), \mathrm{FeOH}^{+1}\left(\mathrm{~N}_{10}, \mathrm{n}_{10}\right), \mathrm{FeSO}_{4}\left(\mathrm{~N}_{11}, \mathrm{n}_{11}\right)$ $\mathrm{Fe}^{+3}\left(\mathrm{~N}_{12}, \mathrm{n}_{12}\right), \mathrm{FeOH}^{+2}\left(\mathrm{~N}_{13}, \mathrm{n}_{13}\right)$,

$\mathrm{Fe}(\mathrm{OH})_{2}^{+1}\left(\mathrm{~N}_{14}, \mathrm{n}_{14}\right), \mathrm{Fe}_{2}(\mathrm{OH})_{2}^{+4}\left(\mathrm{~N}_{15}, \mathrm{n}_{15}\right) ; \mathrm{FeSO}_{4}^{+1}\left(\mathrm{~N}_{16}, \mathrm{n}_{16}\right)$ $\mathrm{Fe}\left(\mathrm{SO}_{4}\right)_{2}^{-1}\left(\mathrm{~N}_{17}, \mathrm{n}_{17}\right), \mathrm{K}^{+1}\left(\mathrm{~N}_{18}, \mathrm{n}_{18}\right)$,

$\mathrm{MnO}_{4}^{-1}\left(\mathrm{~N}_{19}, \mathrm{n}_{19}\right), \mathrm{MnO}_{4}^{-2}\left(\mathrm{~N}_{20}, \mathrm{n}_{20}\right), \mathrm{Mn}^{+3}\left(\mathrm{~N}_{21}, \mathrm{n}_{21}\right), \mathrm{MnOH}^{+2}$ $\left(\mathrm{N}_{22}, \mathrm{n}_{22}\right), \mathrm{Mn}^{+2}\left(\mathrm{~N}_{23}, \mathrm{n}_{23}\right), \mathrm{MnOH}^{+1}\left(\mathrm{~N}_{24}, \mathrm{n}_{24}\right), \mathrm{MnSO}_{4}\left(\mathrm{~N}_{25}, \mathrm{n}_{25}\right)$

The presence of $\mathrm{CO}_{2}$ in $\mathrm{T}$ and $\mathrm{D}$ is considered here as an 
admixture from air, to imitate real conditions of the analysis, on the step of preparation of $\mathrm{D}$ and $\mathrm{T}$; the titration $\mathrm{T}(\mathrm{V}) \Rightarrow \mathrm{D}(\mathrm{V}$ $\left.{ }_{0}\right)$ is realized in the closed system, under isothermal conditions. Precipitation of $\mathrm{MnO}_{2}$ does not occur at sufficiently low $\mathrm{pH}$ value [1].

The $\mathrm{D}+\mathrm{T}$ dynamic redox system is then composed of nonredox static subsystems: D and T. On this basis, some general properties involved with non-redox and redox systems will be indicated. Different forms of GEB, resulting from linear combinations of charge and elemental balances related to D+T system, will be obtained.

To avoid (possible) disturbances, the common notation (subscripts) assumed in the set (14) of species will be applied for components and species in $\mathrm{T}, \mathrm{D}$ and $\mathrm{D}+\mathrm{T}$. In context with the dynamic $\mathrm{D}+\mathrm{T}$ system, $\mathrm{T}$ and $\mathrm{D}$ are considered as static (sub) systems.

\section{Linear combination of balances}

\section{The T subsystem:}

We get here the balances:

$f_{0}=\mathrm{ChB}:$

$\mathrm{N}_{2}-\mathrm{N}_{3}-\mathrm{N}_{7}-2 \mathrm{~N}_{8}+\mathrm{N}_{18}-\mathrm{N}_{19}=0$

$f_{1}=f(\mathrm{H})$ :

$2 \mathrm{~N}_{1}+\mathrm{N}_{2}\left(1+2 \mathrm{n}_{2}\right)+\mathrm{N}_{3}\left(1+2 \mathrm{n}_{3}\right)+\mathrm{N}_{6}\left(2+2 \mathrm{n}_{6}\right)+\mathrm{N}_{7}\left(1+2 \mathrm{n}_{7}\right)+2 \mathrm{~N}_{8} \mathrm{n}_{8}$ $+2 \mathrm{~N}_{18} \mathrm{n}_{18}+2 \mathrm{~N}_{19} \mathrm{n}_{19}=2 \mathrm{~N}_{02}$

$f_{2}=f(0)$ :

$\mathrm{N}_{1}+\mathrm{N}_{2} \mathrm{n}_{2}+\mathrm{N}_{3}\left(1+\mathrm{n}_{3}\right)+\mathrm{N}_{6}\left(3+\mathrm{n}_{6}\right)+\mathrm{N}_{7}\left(3+\mathrm{n}_{7}\right)+\mathrm{N}_{8}\left(3+\mathrm{n}_{8}\right)+$ $\mathrm{N}_{18} \mathrm{n}_{18}+\mathrm{N}_{19}\left(4+\mathrm{n}_{19}\right)$

$=4 \mathrm{~N}_{01}+\mathrm{N}_{02}+2 \mathrm{~N}_{03}$

$-4 \cdot f_{3}=-4 \cdot f\left(\mathrm{CO}_{3}\right):$

$4 \mathrm{~N}_{03}=4 \mathrm{~N}_{6}+4 \mathrm{~N}_{7}+4 \mathrm{~N}_{8}$

$-f_{4}=-f(K):$

$\mathrm{N}_{01}=\mathrm{N}_{18}$

$-7 \cdot f_{5}=-7 \cdot f(\mathrm{Mn}):$

$7 \mathrm{~N}_{01}=7 \mathrm{~N}_{19}$

$f_{12}=2 f_{2}-f_{1}=2 f(\mathrm{O})-f(\mathrm{H})$

$-\mathrm{N}_{2}+\mathrm{N}_{3}+4 \mathrm{~N}_{6}+5 \mathrm{~N}_{7}+6 \mathrm{~N}_{8}+8 \mathrm{~N}_{19}=8 \mathrm{~N}_{01}+4 \mathrm{~N}_{03}$

$f_{12}+f_{0}-4 f_{3}-f_{4}-7 f_{5}=0 \Leftrightarrow(+1) \cdot f_{1}+(-2) \cdot f_{2}+(+4) \cdot f_{3}+(+1) \cdot f_{4}$ $+(+7) \cdot f_{5}-f_{0}=0 \Leftrightarrow$

$(+1) \cdot f(\mathrm{H})+(-2) \cdot f(\mathrm{O})+(+4) \cdot f\left(\mathrm{CO}_{3}\right)+(+1) \cdot f(\mathrm{~K})+(+7) \cdot f(\mathrm{Mn})-$ $\mathrm{ChB}=0$ i.e., the the linear combination (15) is transformed into identity, $0=0$. The coefficients/multipliers at the balances $f\left(\mathrm{Y}_{\mathrm{k}}\right)$ are equal to ONs of elements in the corresponding species.

\section{The D subsystem:}

We get here the balances:

$f_{0}=\mathrm{ChB}$

$\mathrm{N}_{2}-\mathrm{N}_{3}-\mathrm{N}_{4}-2 \mathrm{~N}_{5}-\mathrm{N}_{7}-2 \mathrm{~N}_{8}+2 \mathrm{~N}_{9}+\mathrm{N}_{10}=0$

$f_{1}=f(\mathrm{H})$

$2 \mathrm{~N}_{1}+\mathrm{N}_{2}\left(1+2 \mathrm{n}_{2}\right)+\mathrm{N}_{3}\left(1+2 \mathrm{n}_{3}\right)+\mathrm{N}_{4}\left(1+2 \mathrm{n}_{4}\right)+2 \mathrm{~N}_{5} \mathrm{n}_{5}+\mathrm{N}_{6}\left(2+2 \mathrm{n}_{6}\right)$ $+\mathrm{N}_{7}\left(1+2 \mathrm{n}_{7}\right)+2 \mathrm{~N}_{8} \mathrm{n}_{8}+2 \mathrm{~N}_{9} \mathrm{n}_{9}+$

$\mathrm{N}_{10}\left(1+2 \mathrm{n}_{10}\right)+2 \mathrm{~N}_{11} \mathrm{n}_{11}=14 \mathrm{~N}_{04}+2 \mathrm{~N}_{05}+2 \mathrm{~N}_{06}$

$f_{2}=f(0)$

$\mathrm{N}_{1}+\mathrm{N}_{2} \mathrm{n}_{2}+\mathrm{N}_{3}\left(1+\mathrm{n}_{3}\right)+\mathrm{N}_{4}\left(4+\mathrm{n}_{4}\right)+\mathrm{N}_{5}\left(4+\mathrm{n}_{5}\right)+\mathrm{N}_{6}\left(3+\mathrm{n}_{6}\right)+$ $\mathrm{N}_{7}\left(3+\mathrm{n}_{7}\right)+\mathrm{N}_{8}\left(3+\mathrm{n}_{8}\right)+\mathrm{N}_{9} \mathrm{n}_{9}+$

$\mathrm{N}_{10}\left(1+\mathrm{n}_{10}\right)+\mathrm{N}_{11}\left(4+\mathrm{n}_{11}\right)=11 \mathrm{~N}_{04}+4 \mathrm{~N}_{05}+\mathrm{N}_{06}+2 \mathrm{~N}_{07}$

$-4 \cdot f_{3}=-4 \cdot f\left(\mathrm{CO}_{3}\right)$

$4 \mathrm{~N}_{07}=4 \mathrm{~N}_{6}+4 \mathrm{~N}_{7}+4 \mathrm{~N}_{8}$

$-6 \cdot f_{6}=-6 \cdot f\left(\mathrm{SO}_{4}\right)$

$6 \mathrm{~N}_{04}+6 \mathrm{~N}_{05}=6 \mathrm{~N}_{4}+6 \mathrm{~N}_{5}+6 \mathrm{~N}_{11}$

$-2 \cdot f_{7}=-2 \cdot f(\mathrm{Fe})$

$2 \mathrm{~N}_{04}=2 \mathrm{~N}_{9}+2 \mathrm{~N}_{10}+2 \mathrm{~N}_{11}$

$f_{12}+f_{0}-4 \cdot f_{3}-6 \cdot f_{6}-2 \cdot f_{7}$

$0=0$

i.e., the the linear combination (16) is transformed into identity, $0=0$. From transformation of (16)

$$
\begin{aligned}
& (+1) \cdot f_{1}+(-2) \cdot f_{2}+(+4) \cdot f_{3}+(+6) \cdot f_{6}+(+2) \cdot f_{7}-f_{0} \Rightarrow \\
& (+1) \cdot f(\mathrm{H})+(-2) \cdot f(0)+(+4) \cdot f\left(\mathrm{CO}_{3}\right)+(+6) \cdot f\left(\mathrm{SO}_{4}\right)+(+2) \cdot f(\mathrm{Fe})-
\end{aligned}
$$
$\mathrm{ChB}$

we see again that the coefficients/multipliers at the balances $f\left(Y_{\mathrm{k}}\right)$ are equal to ONs of elements in the corresponding species.

\section{The $\mathrm{D}+\mathrm{T}$ system:}

For the D+T system, from (14) we have the balances:

$f_{0}=\mathrm{ChB}$

$\mathrm{N}_{2}-\mathrm{N}_{3}-\mathrm{N}_{4}-2 \mathrm{~N}_{5}-\mathrm{N}_{7}-2 \mathrm{~N}_{8}+2 \mathrm{~N}_{9}+\mathrm{N}_{10}+3 \mathrm{~N}_{12}+2 \mathrm{~N}_{13}+\mathrm{N}_{14}$ $+4 \mathrm{~N}_{15}+\mathrm{N}_{16}-\mathrm{N}_{17}+\mathrm{N}_{18}-\mathrm{N}_{19}-2 \mathrm{~N}_{20}+$

$3 \mathrm{~N}_{21}+2 \mathrm{~N}_{22}+2 \mathrm{~N}_{23}+\mathrm{N}_{24}=0$

$f_{1}=f(H)$

$2 \mathrm{~N}_{1}+\mathrm{N}_{2}\left(1+2 \mathrm{n}_{2}\right)+\mathrm{N}_{3}\left(1+2 \mathrm{n}_{3}\right)+\mathrm{N}_{4}\left(1+2 \mathrm{n}_{4}\right)+2 \mathrm{~N}_{5} \mathrm{n}_{5}+\mathrm{N}_{6}\left(2+2 \mathrm{n}_{6}\right)$ 
$+\mathrm{N}_{7}\left(1+2 \mathrm{n}_{7}\right)+2 \mathrm{~N}_{8} \mathrm{n}_{8}+2 \mathrm{~N}_{9} \mathrm{n}_{9}+$

$\mathrm{N}_{10}\left(1+2 \mathrm{n}_{10}\right)+2 \mathrm{~N}_{11} \mathrm{n}_{11}+2 \mathrm{~N}_{12} \mathrm{n}_{12}+\mathrm{N}_{13}\left(1+2 \mathrm{n}_{13}\right)+\mathrm{N}_{14}\left(2+2 \mathrm{n}_{14}\right)+$ $\mathrm{N}_{15}\left(2+2 \mathrm{n}_{15}\right)+2 \mathrm{~N}_{16} \mathrm{n}_{16}+2 \mathrm{~N}_{17} \mathrm{n}_{17}+$

$2 \mathrm{~N}_{18} \mathrm{n}_{18}+2 \mathrm{~N}_{19} \mathrm{n}_{19}+2 \mathrm{~N}_{20} \mathrm{n}_{20}+2 \mathrm{~N}_{21} \mathrm{n}_{21}+\mathrm{N}_{22}\left(1+2 \mathrm{n}_{22}\right)+2 \mathrm{~N}_{23} \mathrm{n}_{23}+$ $\mathrm{N}_{24}\left(1+2 \mathrm{n}_{24}\right)+2 \mathrm{~N}_{25} \mathrm{n}_{25}$

$=2 \mathrm{~N}_{02}+14 \mathrm{~N}_{04}+2 \mathrm{~N}_{05}+2 \mathrm{~N}_{06}$

$f_{2}=f(0)$

$\mathrm{N}_{1}+\mathrm{N}_{2} \mathrm{n}_{2}+\mathrm{N}_{3}\left(1+\mathrm{n}_{3}\right)+\mathrm{N}_{4}\left(4+\mathrm{n}_{4}\right)+\mathrm{N}_{5}\left(4+\mathrm{n}_{5}\right)+\mathrm{N}_{6}\left(3+\mathrm{n}_{6}\right)+$ $\mathrm{N}_{7}\left(3+\mathrm{n}_{7}\right)+\mathrm{N}_{8}\left(3+\mathrm{n}_{8}\right)+\mathrm{N}_{9} \mathrm{n}_{9}+$

$\mathrm{N}_{10}\left(1+\mathrm{n}_{10}\right)+\mathrm{N}_{11}\left(4+\mathrm{n}_{11}\right)+\mathrm{N}_{12} \mathrm{n}_{12}+\mathrm{N}_{13}\left(1+\mathrm{n}_{13}\right)+\mathrm{N}_{14}\left(2+\mathrm{n}_{14}\right)+$ $\mathrm{N}_{15}\left(2+\mathrm{n}_{15}\right)+\mathrm{N}_{16}\left(4+\mathrm{n}_{16}\right)+\mathrm{N}_{17}\left(8+\mathrm{n}_{17}\right)+$

$\mathrm{N}_{18} \mathrm{n}_{18}+\mathrm{N}_{19}\left(4+\mathrm{n}_{19}\right)+\mathrm{N}_{20}\left(4+\mathrm{n}_{20}\right)+\mathrm{N}_{21} \mathrm{n}_{21}+\mathrm{N}_{22}\left(1+\mathrm{n}_{22}\right)+\mathrm{N}_{23} \mathrm{n}_{23}$ $+\mathrm{N}_{24}\left(1+\mathrm{n}_{24}\right)+\mathrm{N}_{25}\left(4+\mathrm{n}_{25}\right)$

$=4 \mathrm{~N}_{01}+\mathrm{N}_{02}+2 \mathrm{~N}_{03}+11 \mathrm{~N}_{04}+4 \mathrm{~N}_{05}+\mathrm{N}_{06}+2 \mathrm{~N}_{07}$

$-4 \cdot f_{3}=-4 \cdot f\left(\mathrm{CO}_{3}\right)$

$4 \mathrm{~N}_{03}+4 \mathrm{~N}_{07}=4 \mathrm{~N}_{6}+4 \mathrm{~N}_{7}+4 \mathrm{~N}_{8}$

$-f_{4}=-f(\mathrm{~K})$ :

$\mathrm{N}_{01}=\mathrm{N}_{18}$

$-f_{5}=-f(\mathrm{Mn})$

(21)

$\mathrm{N}_{01}=\mathrm{N}_{19}+\mathrm{N}_{20}+\mathrm{N}_{21}+\mathrm{N}_{22}+\mathrm{N}_{23}+\mathrm{N}_{24}+\mathrm{N}_{25}$

$-6 \cdot f_{6}=-6 \cdot f\left(\mathrm{SO}_{4}\right)$

$6 \mathrm{~N}_{04}+6 \mathrm{~N}_{05}=6 \mathrm{~N}_{4}+6 \mathrm{~N}_{5}+6 \mathrm{~N}_{11}+6 \mathrm{~N}_{16}+12 \mathrm{~N}_{17}+6 \mathrm{~N}_{25}$

$-f_{7}=-f(\mathrm{Fe})$

$\mathrm{N}_{04}=\mathrm{N}_{9}+\mathrm{N}_{10}+\mathrm{N}_{11}+\mathrm{N}_{12}+\mathrm{N}_{13}+\mathrm{N}_{14}+2 \mathrm{~N}_{15}+\mathrm{N}_{16}+\mathrm{N}_{17}$

Then we have, by turns,

$f_{12}=2 \cdot f(\mathrm{O})-f(\mathrm{H}):$

$-\mathrm{N}_{2}+\mathrm{N}_{3}+7 \mathrm{~N}_{4}+8 \mathrm{~N}_{5}+4 \mathrm{~N}_{6}+5 \mathrm{~N}_{7}+6 \mathrm{~N}_{8}+\mathrm{N}_{10}+8 \mathrm{~N}_{11}+\mathrm{N}_{13}+$ $2 \mathrm{~N}_{14}+2 \mathrm{~N}_{15}+8 \mathrm{~N}_{16}+16 \mathrm{~N}_{17}+$

$8 \mathrm{~N}_{19}+8 \mathrm{~N}_{20}+\mathrm{N}_{22}+\mathrm{N}_{24}+8 \mathrm{~N}_{25}=8 \mathrm{~N}_{01}+4 \mathrm{~N}_{03}+8 \mathrm{~N}_{04}+$ $6 \mathrm{~N}_{05}+4 \mathrm{~N}_{07}$

(25)

(26)

$$
f_{12}+f_{0}-4 \cdot f_{3}-f_{4}-6 \cdot f_{6}
$$

$2\left(\mathrm{~N}_{9}+\mathrm{N}_{10}+\mathrm{N}_{11}\right)+3\left(\mathrm{~N}_{12}+\mathrm{N}_{13}+\mathrm{N}_{14}+2 \mathrm{~N}_{15}+\mathrm{N}_{16}+\mathrm{N}_{17}\right)+7 \mathrm{~N}_{19}+6 \mathrm{~N}_{20}+$ $3\left(\mathrm{~N}_{21}+\mathrm{N}_{22}\right)+$

$2\left(\mathrm{~N}_{23}+\mathrm{N}_{24}+\mathrm{N}_{25}\right)=7 \mathrm{~N}_{01}+2 \mathrm{~N}_{04}$

From transformation of (26) we have

$$
(+1) \cdot f_{1}+(-2) \cdot f_{2}+(+4) \cdot f_{3}+(+1) \cdot f_{4}+(+6) \cdot f_{6}-f_{0} \quad \Rightarrow
$$

$(+1) \cdot f(\mathrm{H})+(-2) \cdot f(\mathrm{O})+(+4) \cdot f\left(\mathrm{CO}_{3}\right)+(+1) \cdot f(\mathrm{~K})+(+6) \cdot f\left(\mathrm{SO}_{4}\right)-$ ChB

We see that the coefficients/multipliers at the related balances $f_{\mathrm{k}}(\mathrm{k}=1,2,3,4,6)$ are equal to ONs of elements in the corresponding species; the balances $f_{\mathrm{k}}$ in (28) are related to fans.

Applying the relations:

$\left[\mathrm{X}_{\mathrm{i}}^{\mathrm{zi}}\right] \cdot\left(\mathrm{V}_{\mathrm{o}}+\mathrm{V}\right)=10^{3} \cdot \mathrm{N}_{\mathrm{i}} / \mathrm{N}_{\mathrm{A}}, \mathrm{CV}=10^{3} \cdot \mathrm{N}_{\mathrm{o1}} / \mathrm{N}_{\mathrm{A}}, \mathrm{C}_{0} \mathrm{~V}_{\mathrm{o}}=10^{3} \cdot \mathrm{N}_{04} /$ $\mathrm{N}_{\mathrm{A}}, \mathrm{C}_{1} \mathrm{~V}=10^{3 \cdot} \cdot \mathrm{N}_{03} / \mathrm{N}_{\mathrm{A}}$,

$$
\mathrm{C}_{01} \mathrm{~V}_{\mathrm{o}}=10^{3} \cdot \mathrm{N}_{05} / \mathrm{N}_{\mathrm{A}}, \mathrm{C}_{02} \mathrm{~V}_{\mathrm{o}}=10^{3} \cdot \mathrm{N}_{07} / \mathrm{N}_{\mathrm{A}}
$$

we rewrite Eq. (26) in the more explicit form as

$2\left(\left[\mathrm{Fe}^{+2}\right]+\left[\mathrm{FeOH}^{+1}\right]+\left[\mathrm{FeSO}_{4}\right]\right)+3\left(\left[\mathrm{Fe}^{+3}\right]+\left[\mathrm{FeOH}^{+2}\right]+\left[\mathrm{Fe}(\mathrm{OH})_{2}^{+1}\right]\right.$ $\left.+2\left[\mathrm{Fe}_{2}(\mathrm{OH})_{2}{ }^{+4}\right]+\left[\mathrm{FeSO}_{4}^{+1}\right]+\left[\mathrm{Fe}\left(\mathrm{SO}_{4}\right)_{2}^{-1}\right]\right)$

$+7\left[\mathrm{MnO}_{4}^{-1}\right]+6\left[\mathrm{MnO}_{4}^{-2}\right]+3\left(\left[\mathrm{Mn}^{+3}\right]+\left[\mathrm{MnOH}^{+2}\right]\right)+$ $2\left(\left[\mathrm{Mn}^{+2}\right]+\left[\mathrm{MnOH}^{+1}\right]+\left[\mathrm{MnSO}_{4}\right]\right)$

$$
=\left(7 \mathrm{CV}+2 \mathrm{C}_{0} \mathrm{~V}_{0}\right) /\left(\mathrm{V}_{0}+\mathrm{V}\right)
$$

Eq. (30), obtained from $(25,28,29)$, consists only of the species, where players are involved. Other linear combinations were also used. Applying atomic numbers: $\mathrm{Z}_{\mathrm{Mn}}=25$ and $\mathrm{Z}_{\mathrm{Fe}}=$ 26, we have

$$
\mathrm{Z}_{\mathrm{Fe}} \cdot f_{7}+\mathrm{Z}_{\mathrm{Mn}} \cdot f_{5}-\left(f_{12}+f_{0}-4 \cdot f_{3}-f_{4}-6 \cdot f_{6}\right):
$$

$\left(\mathrm{Z}_{\mathrm{Fe}}-2\right) \cdot\left(\mathrm{N}_{9}+\mathrm{N}_{10}+\mathrm{N}_{11}\right)+\left(\mathrm{Z}_{\mathrm{Fe}}-3\right) \cdot\left(\mathrm{N}_{12}+\mathrm{N}_{13}+\mathrm{N}_{14}+2 \mathrm{~N}_{15}+\mathrm{N}_{16}+\mathrm{N}_{17}\right)+$ $\left(Z_{\mathrm{Mn}}-7\right) \cdot \mathrm{N}_{19}+\left(\mathrm{Z}_{\mathrm{Mn}}-6\right) \cdot \mathrm{N}_{20}$

$+\left(\mathrm{Z}_{\mathrm{Mn}}-3\right) \cdot\left(\mathrm{N}_{21}+\mathrm{N}_{22}\right)+\left(\mathrm{Z}_{\mathrm{Mn}}-2\right) \cdot\left(\mathrm{N}_{23}+\mathrm{N}_{24}+\mathrm{N}_{25}\right)=\left(\mathrm{Z}_{\mathrm{Fe}}-2\right) \cdot \mathrm{N}_{04}+$ $\left(\mathrm{Z}_{\mathrm{Mn}}-7\right) \cdot \mathrm{N}_{01} \quad \Rightarrow$

$\left(\mathrm{Z}_{\mathrm{Fe}}-2\right) \cdot\left(\left[\mathrm{Fe}^{+2}\right]+\left[\mathrm{FeOH}^{+1}\right]+\left[\mathrm{FeSO}_{4}\right]\right)+\left(\mathrm{Z}_{\mathrm{Fe}}-3\right) \cdot\left(\left[\mathrm{Fe}^{+3}\right]+\right.$ $\left.\left[\mathrm{FeOH}^{+2}\right]+\left[\mathrm{Fe}(\mathrm{OH})_{2}{ }^{+1}\right]+2\left[\mathrm{Fe}_{2}(\mathrm{OH})_{2}{ }^{+4}\right]+\left[\mathrm{FeSO}_{4}{ }_{4}^{+1}\right]+\left[\mathrm{Fe}\left(\mathrm{SO}_{4}\right)_{2}{ }^{-1}\right]\right)$ $+\left(\mathrm{Z}_{\mathrm{Mn}}-7\right) \cdot\left[\mathrm{MnO}_{4}^{-1}\right]+\left(\mathrm{Z}_{\mathrm{Mn}}-6\right) \cdot\left[\mathrm{MnO}_{4}^{-2}\right]+\left(\mathrm{Z}_{\mathrm{Mn}}-3\right) \cdot\left(\left[\mathrm{Mn}^{+3}\right]+\right.$ $\left.\left[\mathrm{MnOH}^{+2}\right]\right)+$

$\left(\mathrm{Z}_{\mathrm{Mn}}-2\right) \cdot\left(\left[\mathrm{Mn}^{+2}\right]+\left[\mathrm{MnOH}^{+1}\right]+\left[\mathrm{MnSO}_{4}\right]\right)=\left(\left(\mathrm{Z}_{\mathrm{Fe}}-2\right) \cdot \mathrm{C}_{0} \mathrm{~V}_{\mathrm{o}}+\right.$ $\left.\left(\mathrm{Z}_{\mathrm{Mn}}-7\right) \cdot \mathrm{CV}\right) /\left(\mathrm{V}_{\mathrm{o}}+\mathrm{V}\right)$

Equation (32) results innediately from the Approach I to GEB, see [6].

The least extended (the most compact) form is as follows $[8,25]$

$$
\begin{aligned}
& 3 \cdot f_{7}+2 \cdot f_{5}-\left(f_{12}+f_{0}-4 \cdot f_{3}-f_{4}-6 \cdot f_{6}\right): \\
& \left(N_{9}+N_{10}+N_{11}\right)-\left(5 N_{19}+4 N_{20}+N_{21}+N_{22}\right)=N_{04}-5 N_{01}
\end{aligned}
$$

$\left[\mathrm{Fe}^{+2}\right]+\left[\mathrm{FeOH}^{+1}\right]+\left[\mathrm{FeSO}_{4}\right]-\left(5\left[\mathrm{MnO}_{4}^{-1}\right]+4\left[\mathrm{MnO}_{4}^{-2}\right]+\right.$ $\left.\left[\mathrm{Mn}^{+3}\right]+\left[\mathrm{MnOH}^{+2}\right]\right)$

$$
=\left(\mathrm{C}_{0} \mathrm{~V}_{\mathrm{o}}-5 \cdot \mathrm{CV}\right) /\left(\mathrm{V}_{\mathrm{o}}+\mathrm{V}\right)
$$

Eq. (25) for $f_{12}$ (24), considered as pr-GEB (Eq. 1), can also be rewritten in terms of concentrations; we have 
$-\left[\mathrm{H}^{+1}\right]+\left[\mathrm{OH}^{-1}\right]+7\left[\mathrm{HSO}_{4}^{-1}\right]+8\left[\mathrm{SO}_{4}^{-2}\right]+4\left[\mathrm{H}_{2} \mathrm{CO}_{3}\right]+5\left[\mathrm{HCO}_{3}^{-1}\right]$ $+6\left[\mathrm{CO}_{3}^{-2}\right]+\left[\mathrm{FeOH}^{+1}\right]+8\left[\mathrm{FeSO}_{4}\right]+$

$\left[\mathrm{FeOH}^{+2}\right]+2\left[\mathrm{Fe}(\mathrm{OH})_{2}{ }^{+1}\right]+8\left[\mathrm{Fe}\left(\mathrm{SO}_{4}{ }^{+1}\right]+16\left[\mathrm{Fe}\left(\mathrm{SO}_{4}\right)_{2}{ }^{-1}\right]+\right.$ $8\left[\mathrm{MnO}_{4}^{-1}\right]+8\left[\mathrm{MnO}_{4}^{-2}\right]+\left[\mathrm{MnOH}^{+2}\right]+\left[\mathrm{MnOH}^{+1}\right]$ $\left(\mathrm{V}_{0}+\mathrm{V}\right)$

$+8\left[\mathrm{MnSO}_{4}\right]=\left(8 \mathrm{CV}+8 \mathrm{C}_{0} \mathrm{~V}_{0}+4 \mathrm{C}_{1} \mathrm{~V}+6 \mathrm{C}_{01} \mathrm{~V}_{0}+4 \mathrm{C}_{02} \mathrm{~V}_{0}\right) /$

Equations 30, $32-34$ are equivalent forms of GEB for this system. Other linear combinations of the balances are also admitted/possible for this purpose; none of them are reduced to the identity $0=0$. However, the shortest Eq. (33), chosen arbitrarily, seems to be the most useful for calculation purposes - for obvious reasons.

A comment. In section 3.1.3, the $\mathrm{T}$ (section 3.1.1) and $\mathrm{D}$ (section 3.1.2) are non-redox subsystems of the redox D+T system (section 3.1.3); this is not the general regularity, of course. In some other systems, $\mathrm{D}$ or $\mathrm{T}$ or both ( $\mathrm{D}$ and $\mathrm{T}$ ) can form redox subsystems. For example, the $\mathrm{Br}_{2}$ solution considered in $[5,6,37,38]$ is the redox subsystem $D ; I_{2}+K I$ solution is the redox subystem $\mathrm{T}$ in [39].

\section{Calculation procedure for the $\mathrm{D}+\mathrm{T}$ system}

\section{Completing the set of independent balances}

Equation 33 is completed by charge and concentration balances, obtained from Equations 18, 19, 21, 22, 23 and relations (29). We have, by turns,

$\left[\mathrm{H}^{+1}\right]-\left[\mathrm{OH}^{-1}\right]-\left[\mathrm{HSO}_{4}^{-1}\right]-2\left[\mathrm{SO}_{4}^{-2}\right]-\left[\mathrm{HCO}_{3}^{-1}\right]-2\left[\mathrm{CO}_{3}^{-}\right.$ $\left.{ }^{2}\right]+2\left[\mathrm{Fe}^{+2}\right]+\left[\mathrm{FeOH}^{+1}\right]+3\left[\mathrm{Fe}^{+3}\right]+2\left[\mathrm{FeOH}^{+2}\right]+\left[\mathrm{Fe}(\mathrm{OH})_{2}{ }^{+1}\right]+$ $4\left[\mathrm{Fe}_{2}(\mathrm{OH})_{2}{ }^{+4}\right]+\left[\mathrm{FeSO}_{4}{ }^{+1}\right]-\left[\mathrm{Fe}\left(\mathrm{SO}_{4}\right)_{2}{ }^{-1}\right]+\left[\mathrm{K}^{+1}\right]-\left[\mathrm{MnO}_{4}{ }^{-1}\right]-$

$2\left[\mathrm{MnO}_{4}^{-2}\right]+3\left[\mathrm{Mn}^{+3}\right]+2\left[\mathrm{MnOH}^{+2}\right]+2\left[\mathrm{Mn}^{+2}\right]+\left[\mathrm{MnOH}^{+1}\right]=$

$\left[\mathrm{H}_{2} \mathrm{CO}_{3}\right]+\left[\mathrm{HCO}_{3}{ }^{-1}\right]+\left[\mathrm{CO}_{3}^{-2}\right]-\left(\mathrm{C}_{02} \mathrm{~V}_{0}+\mathrm{C}_{1} \mathrm{~V}\right) /\left(\mathrm{V}_{0}+\mathrm{V}\right)=0$

(19a)

$\left[\mathrm{MnO}_{4}^{-1}\right]+\left[\mathrm{MnO}_{4}^{-2}\right]+\left[\mathrm{Mn}^{+3}\right]+\left[\mathrm{MnOH}^{+2}\right]+\left[\mathrm{Mn}^{+2}\right]+$ $\left[\mathrm{MnOH}^{+1}\right]+\left[\mathrm{MnSO}_{4}\right]$

(21a)

$-\mathrm{CV} /\left(\mathrm{V}_{0}+\mathrm{V}\right)=0$

$\left[\mathrm{HSO}_{4}^{-1}\right]+\left[\mathrm{SO}_{4}^{-2}\right]+\left[\mathrm{FeSO}_{4}\right]+\left[\mathrm{FeSO}_{4}^{+1}\right]+2\left[\mathrm{Fe}\left(\mathrm{SO}_{4}\right)_{2}^{-1}\right]+$ $\left[\mathrm{MnSO}_{4}\right]$ (22a)

$-\left(\mathrm{C}_{\mathrm{o}}+\mathrm{C}_{01}\right) \mathrm{V}_{\mathrm{o}} /\left(\mathrm{V}_{\mathrm{o}}+\mathrm{V}\right)=0$

$\left[\mathrm{Fe}^{+2}\right]+\left[\mathrm{FeOH}^{+1}\right]+\left[\mathrm{FeSO}_{4}\right]+\left[\mathrm{Fe}^{+3}\right]+\left[\mathrm{FeOH}^{+2}\right]+\left[\mathrm{Fe}(\mathrm{OH})_{2}^{+1}\right]$ $+2\left[\mathrm{Fe}_{2}(\mathrm{OH})_{2}{ }^{+4}\right]+$

$\left[\mathrm{FeSO}_{4}^{+1}\right]+\left[\mathrm{Fe}\left(\mathrm{SO}_{4}\right)_{2}^{-1}\right]-\mathrm{C}_{0} \mathrm{~V}_{0} /\left(\mathrm{V}_{0}+\mathrm{V}\right)=0$

The equality (not equation!)

$$
\left[\mathrm{K}^{+1}\right]=\mathrm{CV} /\left(\mathrm{V}_{\mathrm{o}}+\mathrm{V}\right)
$$

(20a)

can enter immediately Eq. 18a like a number, at defined V-value.

\section{The set of interrelations for equilibrium constants}

Concentrations of the species involved in the set of 6 equations: 33 , 18a, 19a, 21a, 22a, 23a, are compatible with the complete set of equilibrium constants, specified as follows:

$\left[\mathrm{H}^{+1}\right]\left[\mathrm{OH}^{-1}\right]=10^{-14.0} ;\left[\mathrm{HSO}_{4}^{-1}\right]=10^{1.8}\left[\mathrm{H}^{+1}\right]\left[\mathrm{SO}_{4}^{-2}\right] ;\left[\mathrm{H}_{2} \mathrm{CO}_{3}\right]=$ $10^{16.4}\left[\mathrm{H}^{+1}\right]^{2}\left[\mathrm{CO}_{3}^{2}\right]$;

$\left[\mathrm{HCO}_{3}{ }^{-1}\right]=10^{10.1}\left[\mathrm{H}^{+1}\right]\left[\mathrm{CO}_{3}^{-2}\right] ;\left[\mathrm{Fe}^{+3}\right]=\left[\mathrm{Fe}^{+2}\right] \cdot 10^{\mathrm{A}(\mathrm{E}-0.771)} ;\left[\mathrm{FeOH}^{+1}\right]$ $=10^{4.5}\left[\mathrm{Fe}^{+2}\right]\left[\mathrm{OH}^{-1}\right]$;

$\left[\mathrm{FeOH}^{+2}\right]=10^{11.0}\left[\mathrm{Fe}^{+3}\right]\left[\mathrm{OH}^{-1}\right] ;\left[\mathrm{Fe}(\mathrm{OH})_{2}^{+1}\right]=10^{21.7}\left[\mathrm{Fe}^{+3}\right]\left[\mathrm{OH}^{-1}\right]^{2} ;$ $\left[\mathrm{Fe}_{2}(\mathrm{OH})_{2}{ }^{+4}\right]=10^{21.7}\left[\mathrm{Fe}^{+3}\right]^{2}\left[\mathrm{OH}^{-1}\right]^{2}$;

$\left[\mathrm{FeSO}_{4}\right]=10^{2.3}\left[\mathrm{Fe}^{+2}\right]\left[\mathrm{SO}_{4}^{-2}\right] ;\left[\mathrm{FeSO}_{4}^{+1}\right]=10^{4.18}\left[\mathrm{Fe}^{+3}\right]\left[\mathrm{SO}_{4}^{-2}\right] ;$ $\left[\mathrm{Fe}\left(\mathrm{SO}_{4}\right)_{2}^{-1}\right]=10^{7 \cdot 4}\left[\mathrm{Fe}^{+3}\right]\left[\mathrm{SO}_{4}^{-2}\right]^{2}$;

$\left[\mathrm{MnO}_{4}^{-1}\right]=\left[\mathrm{Mn}^{+2}\right] \cdot 10^{5 \mathrm{~A}(\mathrm{E}-1.507)+8 \mathrm{pH}} ;\left[\mathrm{MnO}_{4}^{-2}\right]=\left[\mathrm{Mn}^{+2}\right] \cdot 10^{4 \mathrm{~A}(\mathrm{E}}$ $-1.743)+8 \mathrm{pH} ;\left[\mathrm{Mn}^{+3}\right]=\left[\mathrm{Mn}^{+2}\right] \cdot 10^{\mathrm{A}(\mathrm{E}-1.509)} ;\left[\mathrm{MnOH}^{+2}\right]=10^{14 \cdot 2}\left[\mathrm{Mn}^{+3}\right]$ $\left[\mathrm{OH}^{-1}\right]$

The electrode potentials E [V] are put in context with standard electrode potentials $\mathrm{E}_{\mathrm{oi}}$, expressed in SHE scale [40].

\section{The set of independent variables}

The number of equations is equal to the number of 6 independent variables, chosen as components of the vector:

$$
\mathbf{x}=\left[\mathrm{x}_{1}, \ldots, \mathrm{x}_{6}\right]^{\mathrm{T}}=[\mathrm{E}, \mathrm{pH}, \mathrm{pMn} 2, \mathrm{pFe} 2, \mathrm{pSO} 4, \mathrm{pH} 2 \mathrm{CO} 3]^{\mathrm{T}}
$$

where $\mathrm{E}-$ potential $[\mathrm{V}], \mathrm{pH}=-\log \left[\mathrm{H}^{+1}\right], \mathrm{pMn} 2=$ $\log \left[\mathrm{Mn}^{+2}\right], \mathrm{pFe} 2=-\log \left[\mathrm{Fe}^{+2}\right]$, pSO4 $=-\log \left[\mathrm{SO}_{4}^{-2}\right], \mathrm{pH} 2 \mathrm{CO} 3=$ $-\log \left[\mathrm{H}_{2} \mathrm{CO}_{3}\right]$.

\section{Mole fraction as the parameter of D+T system}

All the variables, put in logarithmic scale, are considered as functions of volume $\mathrm{V}$ of the titrant $\mathrm{T}$ added during the titration $\mathrm{T} \Rightarrow \mathrm{D}, \mathrm{x}_{\mathrm{i}}=\mathrm{x}_{\mathrm{i}}(\mathrm{V})$. The $\mathrm{V}[\mathrm{mL}]$ is considered as parameter of the $\mathrm{D}+\mathrm{T}$ system. On this basis, the mole fraction values

$$
\phi=\frac{\mathrm{C} \cdot \mathrm{V}}{\mathrm{C}_{0} \cdot \mathrm{V}_{0}}
$$

are calculated at pre-assumed $\mathrm{V}_{0}, \mathrm{C}, \mathrm{C}_{0}$ values. The $\Phi$ provides a kind of normalization (independence on $\mathrm{V}_{0}$ value) in the system, and is taken as the independent variable on the abscissa of the related plots. The knowledge of the $\mathrm{x}_{\mathrm{i}}=\mathrm{x}_{\mathrm{i}}(\mathrm{V})$ values, allows also to calculate the concentrations $\mathrm{X}_{\mathrm{i}}^{\mathrm{zi}}$ of the different species of the $\mathrm{D}+\mathrm{T}$ system on the basis of relations (35) and present these changes as plots on the relevant speciation diagrams $\log \mathrm{X}_{\mathrm{i}}^{\mathrm{zi}}=\Theta_{\mathrm{i}}(\Phi)$, together with $\mathrm{E}=\mathrm{E}(\Phi)$ and $\mathrm{pH}=\mathrm{pH}(\Phi)$ relationships. Graphical presentation of the data provides an excellent tool for qualitative and quantitative evaluation of processes, occurring at any point of the titration.

All these data for the $\mathrm{D}+\mathrm{T}$ system are obtained on the basis 
of calculations realized with use of the MATLAB [1] computer program.

\section{Computer program}

function $\mathrm{F}=$ Function_MnO4_Fe(x)

global V Vmin Vstep Vmax Vo C C1 Co C01 C02 H OH fi pH E global Kw pKw A K logK

global $\mathrm{HSO}_{4} \mathrm{SO}_{4} \log \mathrm{HSO}_{4} \operatorname{logSO} 4$

global $\mathrm{H}_{2} \mathrm{CO} 3 \mathrm{HCO}_{3} \mathrm{CO}_{3}$

global $\log \mathrm{H}_{2} \mathrm{CO} 3 \log \mathrm{HCO}_{3} \log \mathrm{CO} 3$

global Mn7O4 Mn604 Mn3 Mn3OH

global logMn7O4 logMn6O4 logMn3 logMn3OH

global Mn2 $\mathrm{Mn} 2 \mathrm{OH} \mathrm{Mn}_{2} \mathrm{SO}_{4}$

global logMn2 logMn2OH logMn2SO4

global Fe2 $\mathrm{Fe} 2 \mathrm{OH}$ Fe2SO4

global logFe2 logFe2OH logFe2SO4

global $\mathrm{Fe} 3 \mathrm{Fe} 3 \mathrm{OH} \mathrm{Fe}_{3} \mathrm{OH} 2 \mathrm{Fe}_{2} \mathrm{OH}_{2} \mathrm{Fe}_{3} \mathrm{SO}_{4} \mathrm{Fe}_{3} \mathrm{SO}_{2}$

global $\log \mathrm{Fe} 3 \log \mathrm{Fe} 3 \mathrm{OH} \log \mathrm{Fe} 3 \mathrm{OH} 2 \mathrm{log} \mathrm{Fe}_{2} \mathrm{OH}_{2}$ logFe3SO4 $\log \mathrm{Fe} 3 \mathrm{SO}_{42}$

$\mathrm{E}=\mathrm{x}(1)$

$\mathrm{pH}=\mathrm{x}(2)$;

$\operatorname{Mn} 2=10 . \wedge-x(3)$

$\mathrm{Fe} 2=10 . \wedge-\mathrm{x}(4)$

$\mathrm{SO} 4=10 . \wedge-\mathrm{x}(5)$

$\mathrm{H} 2 \mathrm{CO} 3=10 . \wedge-\mathrm{x}(6)$;

$\mathrm{H}=10 . \wedge-\mathrm{pH}$

$\mathrm{pKw}=14$;

$\mathrm{Kw}=10 . \wedge-14$

$\mathrm{OH}=\mathrm{Kw} . / \mathrm{H}$;

$A=16.9$;

Mn7O4=Mn2.*10.^(5.*A.*(E-1.507)+8.*pH);

Mn6O4=Mn2.*10.^(4.*A.*(E-1.743)+8.*pH);

Mn3=Mn2.*10.^(A.*(E-1.509));

$\mathrm{Fe} 3=\mathrm{Fe} 2 . * 10 . \wedge(\mathrm{A} . *(\mathrm{E}-0.771))$;

$\mathrm{HSO} 4=10 . \wedge 1.8 . * \mathrm{H} . * \mathrm{SO}_{4}$;

$\mathrm{CO} 3=10 . \wedge-16.4 * \mathrm{H} \wedge-2 * \mathrm{H} 2 \mathrm{CO} 3$
$\mathrm{HCO} 3=10 . \wedge 10.1 * \mathrm{H}^{*} \mathrm{CO} 3$

$\mathrm{Fe} 2 \mathrm{OH}=10 . \wedge 4.5 .{ }^{*} \mathrm{Fe} 2 . * \mathrm{OH}$

$\mathrm{Fe} 2 \mathrm{SO} 4=10 . \wedge 2.3 . * \mathrm{Fe} 2 . * \mathrm{SO}_{4}$

$\mathrm{Fe} 3 \mathrm{OH}=10 . \wedge 11.0 .{ }^{*} \mathrm{Fe} 3 .{ }^{*} \mathrm{OH}$;

$\mathrm{Fe} 3 \mathrm{OH} 2=10 . \wedge 21.7 . * \mathrm{Fe} 3 . * \mathrm{OH} . \wedge 2$;

$\mathrm{Fe} 32 \mathrm{OH} 2=10 . \wedge 25.1 . * \mathrm{Fe} 3 . \wedge 2 . * \mathrm{OH} . \wedge 2$;

$\mathrm{Fe} 3 \mathrm{SO}_{4}=10 . \wedge 4.18 . * \mathrm{Fe} 3 . * \mathrm{SO}$;

$\mathrm{Fe} 3 \mathrm{SO}_{42}=10 . \wedge 7.4 . * \mathrm{Fe} 3 .{ }^{*} \mathrm{SO} 4 . \wedge 2$;

$\mathrm{Mn} 2 \mathrm{OH}=10 . \wedge 3.4 \cdot{ }^{*} \mathrm{Mn} 2 . * \mathrm{OH}$

$\mathrm{Mn} 2 \mathrm{SO} 4=10 . \wedge 2.28 . * \mathrm{Mn} 2 .{ }^{*} \mathrm{SO} 4$

$\mathrm{Mn} 3 \mathrm{OH}=10 . \wedge 14.2 .{ }^{*} \mathrm{Mn} 3 .{ }^{*} \mathrm{OH}$;

$\mathrm{K}=\mathrm{C} . * \mathrm{~V} \cdot /(\mathrm{Vo}+\mathrm{V})$

\%Charge balance

$\mathrm{F}=\left[\left(\mathrm{H}-\mathrm{OH}-\mathrm{HSO}_{4}-2 . * \mathrm{SO}_{4}-\mathrm{HCO} 3-2 \cdot * \mathrm{CO}_{3}-+2 . * \mathrm{Fe} 2+\mathrm{Fe} 2 \mathrm{OH} .\right.\right.$.

+3. $* \mathrm{Fe} 3+2 . * \mathrm{Fe} 3 \mathrm{OH}+\mathrm{Fe} 3 \mathrm{OH} 2+4 . * \mathrm{Fe} 32 \mathrm{OH} 2+\mathrm{Fe} 3 \mathrm{SO}_{4}-$ $\mathrm{Fe} 3 \mathrm{SO} 42 \ldots$

$+\mathrm{K}-\mathrm{Mn} 7 \mathrm{O} 4-2 .{ }^{*} \mathrm{Mn} 6 \mathrm{O} 4+3 * \mathrm{Mn} 3+2 .{ }^{*} \mathrm{Mn} 3 \mathrm{OH}+2 .{ }^{*} \mathrm{Mn} 2+\mathrm{Mn} 2$ $\mathrm{OH})$;

\%Concentration balance of $\mathrm{Mn}$

$\left(\mathrm{Mn} 7 \mathrm{O}_{4}+\mathrm{Mn} 6 \mathrm{O}_{4}+\mathrm{Mn} 3+\mathrm{Mn} 3 \mathrm{OH}+\mathrm{Mn} 2+\mathrm{Mn} 2 \mathrm{OH}+\mathrm{Mn} 2 \mathrm{SO}_{4}-\right.$ C. $*$ V./(Vo+V));

\%Concentration balance of Fe

$\left(\mathrm{Fe} 2+\mathrm{Fe} 2 \mathrm{OH}+\mathrm{Fe} 2 \mathrm{SO}_{4}+\mathrm{Fe} 3+\mathrm{Fe} 3 \mathrm{OH}+\mathrm{Fe} 3 \mathrm{OH} 2+2 .{ }^{*} \mathrm{Fe} 32 \mathrm{OH} 2 \ldots\right.$

+Fe3SO4+Fe3SO42-Co.*Vo./(Vo+V));

\%Concentration balance of $\mathrm{SO}_{4}$

$\left(\mathrm{HSO}_{4}+\mathrm{SO}_{4}+\mathrm{Mn}_{2} \mathrm{SO}_{4}+\mathrm{Fe}_{2} \mathrm{SO}_{4}+\mathrm{Fe}_{3} \mathrm{SO}_{4}+2 \cdot * \mathrm{Fe}_{3} \mathrm{SO}_{4}-\right.$ $(\mathrm{Co}+\mathrm{Ca}) . * \mathrm{Vo} . /(\mathrm{Vo}+\mathrm{V}))$;

\%Concentration balance of $\mathrm{CO} 3$

$\left(\mathrm{H}_{2} \mathrm{CO}_{3}+\mathrm{HCO}_{3}+\mathrm{CO}_{3}-\left(\mathrm{Co}_{2} * \mathrm{Vo}+\mathrm{C} 1 . * \mathrm{~V}\right) /(\mathrm{Vo}+\mathrm{V})\right)$;

\%Electron balance

$\left(\mathrm{Fe} 2+\mathrm{Fe} 2 \mathrm{OH}+\mathrm{Fe}_{2} \mathrm{SO}_{4}-\left(5 \cdot * \mathrm{Mn}_{7} \mathrm{O}_{4}+4 \cdot * \mathrm{Mn} 6 \mathrm{O}_{4}+\right.\right.$ $\mathrm{Mn} 3+\mathrm{Mn} 3 \mathrm{OH}) \ldots$

$-($ Co. $*$ Vo. $-5 . *$ C. $*$ V. $) /(\mathrm{Vo}+\mathrm{V}))]$;

$\log M n 2=\log 10(M n 2)$

$\log \mathrm{Mn} 2 \mathrm{OH}=\log 10(\mathrm{Mn} 2 \mathrm{OH})$;

$\log \mathrm{Mn} 2 \mathrm{SO} 4=\log 10(\mathrm{Mn} 2 \mathrm{SO} 4)$; 
$\log M n 3=\log 10(M n 3)$;

$\log \mathrm{Mn} 3 \mathrm{OH}=\log 10(\mathrm{Mn} 3 \mathrm{OH})$

$\log M n 604=\log 10(M n 604)$;

$\log M n 704=\log 10(M n 704)$;

$\log \mathrm{Fe} 2=\log 10(\mathrm{Fe} 2)$

$\log \mathrm{Fe} 2 \mathrm{OH}=\log 10(\mathrm{Fe} 2 \mathrm{OH})$;

$\log \mathrm{Fe} 2 \mathrm{SO} 4=\log 10\left(\mathrm{Fe} 2 \mathrm{SO}_{4}\right)$;

$\log \mathrm{Fe} 3=\log 10(\mathrm{Fe} 3)$;

$\log \mathrm{Fe} 3 \mathrm{OH}=\log 10(\mathrm{Fe} 3 \mathrm{OH})$;

$\log \mathrm{Fe} 3 \mathrm{OH} 2=\log 10(\mathrm{Fe} 3 \mathrm{OH} 2)$;

$\log \mathrm{Fe} 32 \mathrm{OH} 2=\log 10(\mathrm{Fe} 32 \mathrm{OH} 2)$;

$\log \mathrm{Fe} 3 \mathrm{SO}_{4}=\log 10\left(\mathrm{Fe} 3 \mathrm{SO}_{4}\right)$;

$\log \mathrm{Fe} 3 \mathrm{SO} 42=\log 10(\mathrm{Fe} 3 \mathrm{SO} 42)$;

$\log \mathrm{HSO}_{4}=\log 10\left(\mathrm{HSO}_{4}\right)$

$\log \mathrm{SO}_{4}=\log 10\left(\mathrm{SO}_{4}\right)$

$\log \mathrm{H}_{2} \mathrm{CO} 3=\log 10(\mathrm{H} 2 \mathrm{CO} 3) ;$

$\log \mathrm{HCO}_{3}=\log 10\left(\mathrm{HCO}_{3}\right)$

$\log \mathrm{CO}_{3}=\log 10(\mathrm{CO} 3)$;

$\log \mathrm{K}=\log 10(\mathrm{~K})$;

Graphical presentation of results and discussion

The results of calculations made at $\mathrm{V}_{0}=100, \mathrm{C}_{0}=0.01, \mathrm{C}=0.02$, $\mathrm{C}_{01}=0.5, \mathrm{C}_{02}=\mathrm{C}_{1}=0$ are plotted in Figures 1 and 2 . The jump of $\mathrm{E}$ on the curve in Figure 1a occurs at $\Phi=\Phi_{\mathrm{eq}}=0.2$, i.e., at the equivalent (eq) point where $\mathrm{C} \cdot \mathrm{V}_{\mathrm{eq}}=0.2 \cdot \mathrm{C}_{\mathrm{o}} \cdot \mathrm{V}_{\mathrm{o}}$. Relatively small $\mathrm{pH}$ changes (Figure $1 \mathrm{~b}$ ) result from high buffer capacity of the titrand D [41-46]. From Fig. 2a we see that $\left[\mathrm{Fe}^{+3}\right]<<\left[\mathrm{FeSO}_{4}{ }^{+1}\right]$ $<<\left[\mathrm{Fe}\left(\mathrm{SO}_{4}\right)_{2}^{-1}\right.$. Note that $\mathrm{MnOH}^{+2}$ and $\mathrm{Mn}^{+3}$ (not $\mathrm{MnO}_{4}^{-1}$ ) ions are the predominating manganese species immediately after crossing the related equivalence point (Figure 2b), Some points from the vicinity of equivalence point are presented in Table 1.

Table 1 . The selected pairs $(\Phi, E)$ taken from the vicinity of $\Phi_{\mathrm{eq}}=0.2$ at $\left(\mathrm{V}_{0}, \mathrm{C}_{0}, \mathrm{C}_{01}, \mathrm{C}\right)=(100,0.01,0.5,0.02)$ and $\mathrm{C}_{1}=\mathrm{C}_{02}=0$.

\section{'Variations on a theme'}

Option 1: To indicate a complexation effect of sulphate ions, introduced by the $\mathrm{H}_{2} \mathrm{SO}_{4}$ solution, we compare the plots of $\mathrm{E}=\mathrm{E}(\Phi)$ curves: (1) the curve from Figure 1a with one (2) obtained after omission of the sulfate complexes $\left(\mathrm{FeSO}_{4}\right.$, The related plots are compared in Figure 3.

Figure 3. The $\mathrm{E}=\mathrm{E}()$ relationships: $1-$ as in Figure $1 \mathrm{a} ; 2$ - after omission of sulphate $\mathrm{Fe}$ - and $\mathrm{Mn}$ - complexes in the $\mathrm{FeSO}_{4}^{+1}, \mathrm{Fe}\left(\mathrm{SO}_{4}\right)_{2}^{-1}, \mathrm{MnSO}_{4}$ ) from the algorithm in section 4.5 . related balances; $\mathrm{C}_{0}=0.01, \mathrm{C}_{01}=0.5, \mathrm{C}=0.02$.

Option 2. The sulphate complexes formed by $\mathrm{Mn}^{+3}$ ions are unknown in literature, although on the basis of analogy with other trivalent ions (e.g., $\mathrm{Fe}^{+3}, \mathrm{Al}^{+3}$ ) it could be expected that the stability constants $\mathrm{K}_{\mathrm{i}}$ for virtual $\mathrm{Mn}\left(\mathrm{SO}_{4}\right)_{\mathrm{i}}^{+3-2 i}$ complexes, $\left[\mathrm{Mn}\left(\mathrm{SO}_{4}\right)_{\mathrm{i}}^{+3-2 \mathrm{i}}\right]=\mathrm{K}_{\mathrm{i}} \cdot\left[\mathrm{Mn}^{+3}\right]\left[\mathrm{SO}_{4}^{-2}\right]^{\mathrm{i}}$, may have significant values. However, a numerical analysis of the data obtained for the preassumed stability constants $\mathrm{K}_{i}$ of sulphate complexes (Figure 4) with the curve obtained experimentally [14] has revealed that the $\mathrm{Mn}\left(\mathrm{SO}_{4}\right)_{i}{ }^{+3-2 i}$ complexes - if they exist - are relatively weak [6].

Figure 4. Fragments of hypothetical titration curves plotted for different pairs of stability constants $\left(\mathrm{K}_{1}, \mathrm{~K}_{2}\right)$ of the sulfate complexes $\mathrm{Mn}\left(\mathrm{SO}_{4}\right)_{\mathrm{i}}{ }^{+3-2 \mathrm{i}}: 1-\left(10^{4}, 10^{7}\right), 2-\left(10^{3}, 10^{6}\right), 3-\left(10^{2.5}\right.$, $\left.10^{5}\right), 4-\left(10^{2}, 10^{4}\right), 5-\left(10^{4}, 0\right), 6-\left(10^{3}, 0\right), 7-\left(10^{2}, 0\right), 8-(0$, $0)$, plotted at $\mathrm{C}_{0}=0.01, \mathrm{C}_{01}=0.5, \mathrm{C}=0.02$.

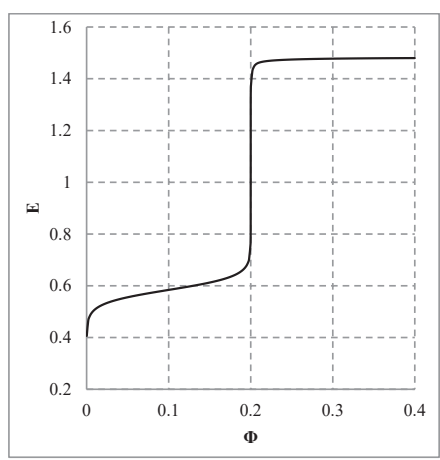

(1a)

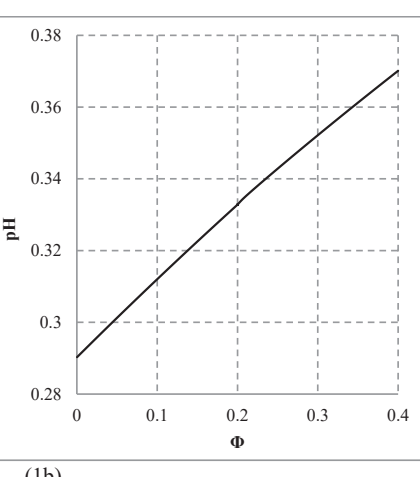

(1b)
Figure 1: The relationships: $(1 \mathrm{a}) \mathrm{E}=\mathrm{E}(\Phi)$ and $(1 \mathrm{~b}) \mathrm{pH}=\mathrm{pH}(\Phi)$ plotted at $\left(\mathrm{V}_{0^{\prime}}, \mathrm{C}_{0}, \mathrm{C}\right.$ $C)=(100,0.01,0.5,0.02)$ and $C_{1}=C_{02}=0$

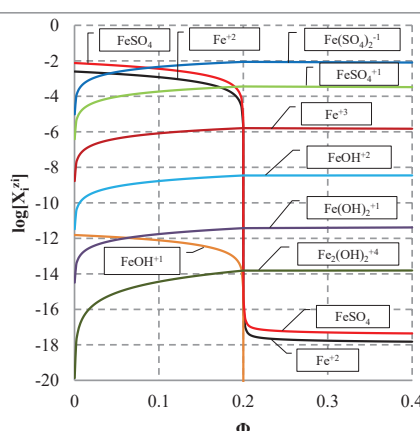

(2a)

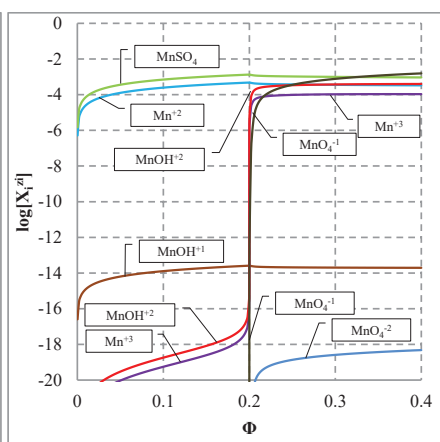

(2b)
Figure 2: System II-3: The speciation curves for (2a) Mn species and (2b) Fe species plotted at $\left(\mathrm{V}_{0}, \mathrm{C}_{0}, \mathrm{C}_{0}, \mathrm{C}\right)=(100,0.01,0.5,0.02)$ and $\mathrm{C}_{1}=\mathrm{C}_{02}=0$.

Table 1: The selected pairs $(\Phi, E)$ taken from the vicinity of $\Phi_{\text {eq }}=0.2$ at $\left(\mathrm{V}_{0}, \mathrm{C}_{0}, \mathrm{C}_{01}\right.$ $C)=(100,0.01,0.5,0.02)$ and $C_{1}=C_{02}=0$

\begin{tabular}{|c|c|}
\hline $\boldsymbol{\Phi}$ & $\mathbf{E}, \mathbf{m V}$ \\
\hline 0.19800 & 0.701 \\
0.19900 & 0.719 \\
0.19980 & 0.761 \\
0.19990 & 0.778 \\
0.19998 & 0.820 \\
0.20000 & 1.034 \\
0.20002 & 1.323 \\
0.20010 & 1.365 \\
0.20020 & 1.382 \\
0.20200 & 1.442 \\
\hline
\end{tabular}

Citation: AM Michałowska-Kaczmarczyk, Michałowski T (2020) Some detailed remarks on elemental and core balances formulated for non-redox and redox electrolytic systems. Biomed Sci Eng 6(1): 021-033. DOI: https://dx.doi.org/10.17352/abse.000017 


\section{Final comments}

The quantitative, algebraic description of any electrolytic system according to GATES principles is based on electroneutrality rule, and on rules of conservation of particular elements in the systems, where none radioactive transformations occur [2]. The electroneutrality rule is expressed by charge balance $\left(f_{0}=\mathrm{ChB}\right)$. The conservation of particular elements is expressed in terms of elemental and core balances $f_{k}=f\left(Y_{k}\right)$, where $Y_{k}(k=1,2, \ldots, K)$ is an element or core. For ordering purposes it is assumed that $\mathrm{Y}_{1}=\mathrm{H}, \mathrm{Y}_{2}=\mathrm{O}$, and the linear combination $f_{12}=2 \cdot f_{2}-f_{1}=2 \cdot f(\mathrm{O})-f(\mathrm{H})$ is formulated. In redox systems, $\mathrm{K}^{*}$ electron-non-active elements/cores ('fans'), and $\mathrm{K}-\mathrm{K}^{*}$ electron-active elements ('players') are distinguished. In all examples presented here, $\mathrm{H}$ and $\mathrm{O}$ did not participated in redox systems as players. The set of $\mathrm{K}$ balances $\left(f_{0}, f_{12}, f_{3}, \ldots, f_{\mathrm{K}}\right)$ is needed for mathematical formulation of a redox system (i.e., at $\mathrm{K}^{*}<\mathrm{K}$ ), whereas the $\mathrm{K}-1$ balances $f_{0}, f_{3}, \ldots, f_{\mathrm{K}}$ are

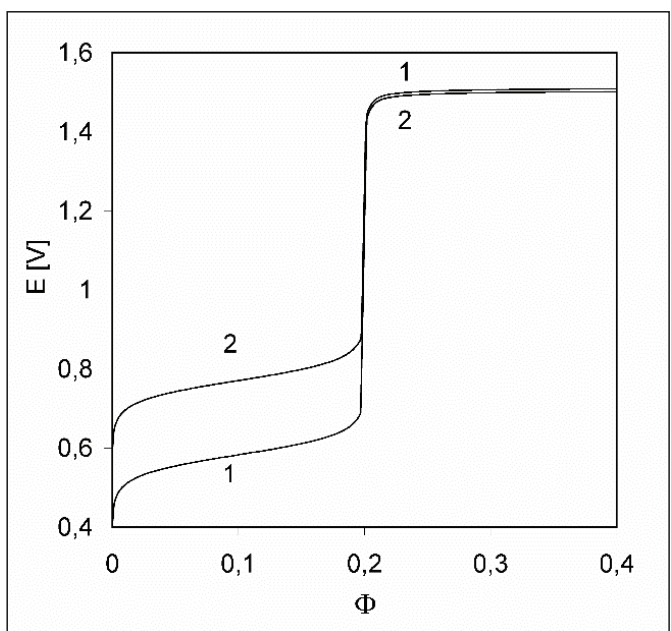

Figure 3: The $E=E(\Phi)$ relationships: 1 - as in Figure 1a; 2 - after omission of sulphate Fe- and $\mathrm{Mn}$ - complexes in the related balances; $\mathrm{C}_{0}=0.01, \mathrm{C}_{01}=0.5, \mathrm{C}=$ 0.02

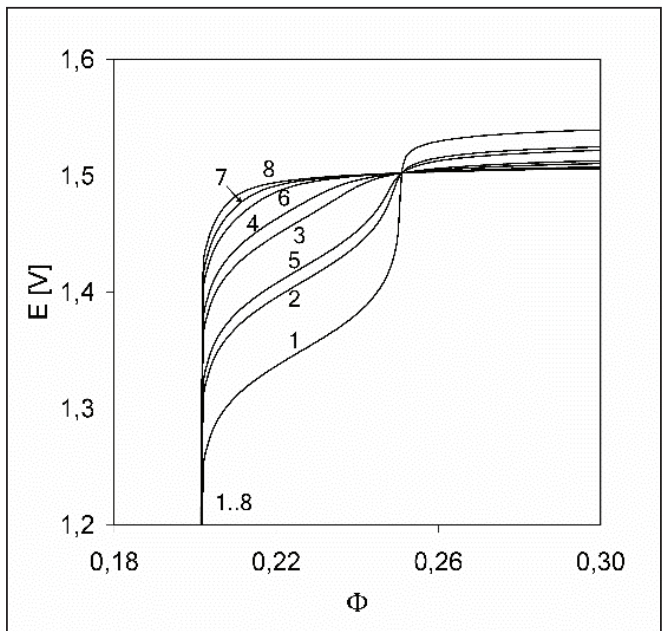

Figure 4: Fragments of hypothetical titration curves plotted for different pairs of stability constants $\left(\mathrm{K}_{1}, \mathrm{~K}_{2}\right)$ of the sulfate complexes $\mathrm{Mn}\left(\mathrm{SO}_{4}\right)_{i}^{+3-2 i}: 1-\left(10^{4}, 10^{7}\right), 2$ $\left(10^{3}, 10^{6}\right), 3-\left(10^{2.5}, 10^{5}\right), 4-\left(10^{2}, 10^{4}\right), 5-\left(10^{4}, 0\right), 6-\left(10^{3}, 0\right), 7-\left(10^{2}, 0\right), 8-(0$, $0)$, plotted at $C_{0}=0.01, C_{01}=0.5, C=0.02$. needed for resolution of a non-redox system, where $\mathrm{K}^{*}=\mathrm{K}$ and $f_{12}$ is the balance linearly dependent on $f_{0}, f_{3}, \ldots, f_{K}$. The linear independency or dependency of $f_{12}$ within the balances $f_{0}, f_{12}, f_{3}, \ldots$ ,$f_{\mathrm{K}}$ is then the general, dichotomous criterion distinguishing between redox and non-redox systems. The linear combination $\sum_{\mathrm{k}=1}^{\mathrm{k}} \mathrm{d}_{\mathrm{k}} \cdot \boldsymbol{f}_{\mathrm{k}}-\boldsymbol{f}_{\mathrm{o}}$ (Eq. 3) applied to a non-redox system $\left(1^{\circ}\right)$ gives the identity, $0=0$, for a non-redox system, or $\left(2^{\circ}\right)$ does not give the identity for a redox system, also after any linear combination with $\mathrm{K}-\mathrm{K}^{*}$ balances for the players in this system. These regularities are valid for coefficients $d_{k}$ equal to oxidation numbers (ONs) of the elements in the corresponding balances $f_{\mathrm{k}}=f\left(\mathrm{Y}_{\mathrm{k}}\right)$ for elements/cores $\mathrm{Y}_{\mathrm{k}}$ related to fans.

The $f_{12}$ and any linear combination of $f_{12}$ with $f_{0}, f_{3}, \ldots, f_{\mathrm{K}}$, have full properties of Generalized Electron Balance (GEB), completing the set of $\mathrm{K}$ balances, $f_{0}, f_{12}, f_{3}, \ldots, f_{\mathrm{K}}$, needed for resolution of a redox system, of any degree of complexity. The supreme role of this independency/dependency criterion, put also in context with calculation of ONs, is of great importance, in context with the contractual nature of the $\mathrm{ON}$ concept known from the literature issued hitherto [47-49]. These regularities are the clear confirmation of the E. Noether's general theorem [50] applied to conservation laws of a physical/electrolytic system, expressed in terms of algebraic equations, where GEB is perceived as the Law of Nature [15], as the hidden connection of physicochemical laws, and as the breakthrough in thermodynamic theory of electrolytic redox systems. Resolution of several redox systems, according to the GATES principles, is presented. The GATES/GEB is the best thermodynamic approach of electrolytic redox systems, of any degree of complexity. The Generalized Equivalent Mass (GEM) concept [14], fully compatible with GATES, was introduced. The mathematical models, applicable for handling the results obtained from potentiometric titrations in redox systems, were also presented. The paper offers - undoubtedly - the best possible ways to resolution of the issues raised. Formulation of an electrolytic redox system specified in section 3.1.3 illustrates the huge possibilities/advantages inherent in GATES/GEB.

All the regularities specified above are valid for redox and non-redox systems of any degree of complexity, also for the multi-solvent systems [51-54].

The key role of $\mathrm{H}$ and $\mathrm{O}$ (not free electrons, $\mathrm{e}^{-1}$ ) in redox systems, inherent in $f_{12}$, was stressed in [23]. Contrary to appearances, established by the current paradigm "obligatory" till now, the criterion distinguishing non-redox and redox systems is not immediately associated with free electrons in the system. The new/ fundamental/practical criterion involved with $f_{12}=2 \cdot f(\mathrm{O})-f(\mathrm{H})$ and its properties, unknown in earlier literature, provides a kind of uniformity in the formulas derived for this purpose. This fact, especially the simple calculations of free electron concentrations in redox systems [23], deny the unique role of free electrons in redox reactions. On the other hand, it points to the decisive role of $\mathrm{H}$ and $\mathrm{O}$ in redox systems [2], suggested elsewhere, in earlier theoretical/ hypothetical considerations on these systems. Here is the hidden simplicity, which had to be discovered, as the Approach II to GEB. The author ${ }^{\mathrm{TM}}$ contends that the discovery of the Approach II GEB would most likely be impossible without the prior discovery of 
the Approach I to GEB. The GEB concept, valid for electrolytic redox systems, is the emanation of balances for $\mathrm{H}$ and $\mathrm{O}$, referred to aqueous media. GEB is compatible with other (charge and concentration) balances and enables to resolve the electrolytic (mono- or/and two-phase) redox systems of any degree of complexity, within the scope of GATES, perceived as the thermodynamic approach to equilibrium and metastable systems, where all necessary physicochemical knowledge on the systems tested is involved. The GATES is perceived as the unrivalled tool applicable, among others: (a) to mathematical modelling of thermodynamic behavior of the systems, (b) in choice of optimal a priori conditions of chemical analyses, and (c) in gaining chemical information invisible in real experiments, in general.

GATES/GEB is a counter-proposal in relation to earlier IUPAC decisions, presented in three subsequent editions of the Orange Book, and based on the reaction stoichiometry; that viewpoint was criticized unequivocally/ exhaustively/ convincingly, especially in a series of authors' articles cited herein. It were demonstrated, on examples of redox systems of different complexity, that stoichiometry is a secondary/ derivative/"fragile" concept, from the viewpoint of GATES, and GATES/GEB, in particular.

Conservation laws of physics are very closely related to the symmetry of physical laws under various transformations. The nature of these connections is an intriguing physical problem. The theory of these connections, as it appears in classical physics, constitutes one of the most beautiful aspects of mathematical physics. It confirms a general theorem of E. Noether which states that symmetries and conservation laws of a physical system correspond to each other [50]. The Noether's conceptual approach to algebra led to a body of principles unifying algebra, geometry, linear algebra, topology, and logic. The theory of this connection constitutes one of the most beautiful chapters of mathematical physics.

Concluding, GATES is the overall, thermodynamic approach to redox and non-redox, static and dynamic, single and multiphase equilibrium, metastable and non-equilibrium electrolytic systems, of any degree of complexity. Possibilities of GATES/GEB are far greater than ones offered by the actual physicochemical knowledge related to the system in question.

Stoichiometry, oxidation number, equivalent mass [14], order of reaction, etc. are derivative (not primary!) concepts within GATES. The Equilibrium Law (EL), based on the Gibbs function and the Lagrange multipliers idea [15], can be put instead of Mass Action Law (MAL), based on a stoichiometric reaction notation, and other principles. Equilibrium, kinetic and metastable systems are distinguished. Within GATES thermodynamics of electrolytic systems is based on purely algebraic principles; the stoichiometry is considered here only as a kind of "dummy" [22,23,55].

Summarizing, this paper offers the best possible ways to resolution of the issues raised.

\section{References}

1. Michałowski T (2011) Application of GATES and MATLAB for Resolution of Equilibrium, Metastable and Non-Equilibrium Electrolytic Systems, Chapter 1 in: Applications of MATLAB in Science and Engineering (ed. by Michałowsk $\mathrm{T})$, InTech - Open Access publisher in the fields of Science, Technology and Medicine 134. Link: https://bit.ly/2YMnNtl

2. Michałowska-Kaczmarczyk AM, Michałowski T (2014) GATES as the Unique Tool for Simulation of Electrolytic Redox and Non-Redox Systems. J Ana Bioanal Tech 5: 204. Link: https://bit.ly/2yFx8bL

3. Michałowska-Kaczmarczyk AM, Michałowski T (2014) Linear Dependence of Balances for Non-Redox Electrolytic Systems. Am J Analyt Chem 5: 1285 1289. Link: https://bit.ly/2WeORzQ

4. Michalowski T (1994) Calculation of $\mathrm{pH}$ and potential $\mathrm{E}$ for bromine aqueous solutions. J Chem Educ 71: 560-562. Link: https://bit.ly/2yFMUmX

5. Michałowski T, Lesiak A (1994) Acid-base titration curves in disproportionating redox systems. J Chem Educ 71: 632-636. Link: https://bit.ly/3blllqS

6. Michałowski T, Lesiak A (1994) Formulation of generalized equations for redox titration curves. Chemia Analityczna (Warsaw) 39: 623-637.

7. Michałowski T, Wajda N, Janecki D (1996) A Unified Quantitative Approach to Electrolytic Systems. Chern Anal (Warsaw) 41: 667-685. Link: https://bit.ly/2LjqXwX

8. Michałowski T, Baterowicz A, Madej A, Kochana J (2001) An extended Gran method and its applicability for simultaneous determination of $\mathrm{Fe}(\mathrm{II})$ and Fe(III). Analytica Chimica Acta 442: 287-293. Link: https://bit.ly/2WCWajS

9. Michałowski T, Rymanowski M, Pietrzyk A (2005) Non-typical Brönsted's acids and bases. J Chem Educ 82: 470-472. Link: https://bit.ly/2WhBJKk

10. Michałowski T, Toporek M, Rymanowski M (2005) Overview on the Gran and other linearization methods applied in titrimetric analyses. Talanta 65: 1241 1253. Link: https://bit.ly/2SLUvXN

11. Michałowski T, Kupiec K, Rymanowski M (2008) Numerical analysis of the Gran methods. A comparative study. Anal Chim Acta 606: 172-183. Link: https://bit.ly/3bgrBFY

12. Ponikvar M, Michałowski T, Kupiec K, Wybraniec S, Rymanowski M (2008) Experimental verification of the modified Gran methods applicable to redox systems. Anal Chim Acta 628: 181-189. Link: https://bit.ly/2LcviSI

13. Michałowski T (2010) The Generalized Approach to Electrolytic Systems: I. Physicochemical and Analytical Implications. Crit Rev Anal Chem 40: 2-16. Link: https://bit.ly/2Ljru1v

14. Michałowski T, Pietrzyk A, Ponikvar-Svet M, Rymanowski M (2010) The Generalized Approach to Electrolytic Systems: II. The Generalized Equivalent Mass (GEM) Concept. Crit Rev Anal Chem 40: 17-29. Link: https://bit.ly/2YNrCi3

15. Michałowska-Kaczmarczyk AM, Spórna-Kucab A, Michałowski T (2017) Generalized Electron Balance (GEB) as the Law of Nature in Electrolytic Redox Systems, in: Redox: Principles and Advanced Applications, Ali Khalid MA (Ed) InTech Chap. 2: 9-55

16. Michałowska-Kaczmarczyk AM, Spórna-Kucab A, Michałowski T (2017) Formulation of simple electrolytic redox systems according to GATES/GEB principles. J Chem Appl Chem Eng 1: 1-10. Link: https://bit.ly/3cu6lxX

17. Michałowska-Kaczmarczyk AM, Michałowski T (2013) Comparative balancing of non-redox and redox electrolytic systems and its consequences. Am J Analyt Chem 4: 46-53. Link: https://bit.ly/2SOCxEe

18. Michałowski T, Toporek M, Michałowska-Kaczmarczyk AM, Asuero AG (2013) New Trends in Studies on Electrolytic Redox Systems. Electrochimica Acta 109: 519-531. Link: https://bit.ly/2znHey5

Citation: AM Michałowska-Kaczmarczyk, Michałowski T (2020) Some detailed remarks on elemental and core balances formulated for non-redox and redox electrolytic systems. Biomed Sci Eng 6(1): 021-033. DOI: https://dx.doi.org/10.17352/abse.000017 
19. Michałowski T, Michałowska-Kaczmarczyk AM, Toporek M (2013) Formulation of general criterion distinguishing between non-redox and redox systems. Electrochimica Acta 112: 199-211. Link: https://bit.ly/2SMInpu

20. Michałowska-Kaczmarczyk AM, Toporek M, Michałowski T (2015) Speciation Diagrams in Dynamic lodide + Dichromate System. Electrochimica Acta 155 217-227. Link: https://bit.ly/2xJYEEq

21. Toporek M, Michałowska-Kaczmarczyk AM, Michałowski T (2015) Symproportionation versus Disproportionation in Bromine Redox Systems. Electrochimica Acta 171: 176-187. Link: https://bit.ly/35K2mKL

22. Michałowska-Kaczmarczyk AM, Asuero AG, Toporek M, Michałowski (2015) "Why not stoichiometry" versus "Stoichiometry - why not?" Part II. GATES in context with redox systems. Crit Rev Anal Chem 45: 240-268. Link: https://bit.ly/3bgf5Gj

23. Michałowska-Kaczmarczyk AM, Michałowski T, Toporek M, Asuero AG (2015) "Why not stoichiometry" versus "Stoichiometry - why not?" Part III, Extension of GATES/GEB on Complex Dynamic Redox Systems. Crit Rev Anal Chem 45: 348-366. Link: https://bit.ly/2WHUy8a

24. Michałowska-Kaczmarczyk AM, Michałowski T, Toporek M (2016) Formulation of Dynamic Redox Systems according to GATES/GEB Principles. Int J Electrochem Sci 11: 2560-2578. Link: https://bit.ly/2LeV08Y

25. Michałowska-Kaczmarczyk AM, Spórna-Kucab A, Michałowski T (2017) A Distinguishing Feature of the Balance 2.f(O) - $f(H)$ in Electrolytic Systems. The Reference to Titrimetric Methods of Analysis, in: Advances in Titration Techniques. Vu Dang Hoang (Ed.), InTech Chap 6: 173-207. Link: https://bit.ly/2SJB1Dp

26. Michałowska-Kaczmarczyk AM, Spórna-Kucab A, Michałowski T (2017) General Properties of the Balances $2 \cdot f(0)-f(H)$ Related to Electrolytic Systems. Anal Chem Ind J 17: 124. Link: https://bit.ly/3dw3kgA

27. Michałowska-Kaczmarczyk AM, Spórna-Kucab A, Michałowski T (2017) Oxidation number, oxidant and reductant as derivative concepts within GATES/ GEB formulation. J Chem Appl Chem Eng 1: 2. Link: https://bit.ly/2zk9e5J

28. Michałowska-Kaczmarczyk AM, Spórna-Kucab A, Michałowski T (2017) Some Regularities Involved with Oxidation Numbers Stated in Formulation of Redox Systems According to GATES/GEB Principles. J Anal Bioanalytic Separation Tech 2: 102-110. Link: https://bit.ly/3dvephK

29. Michałowska-Kaczmarczyk AM, Michałowski T (2018) The Importance of Linear Combinations in Theory of Electrolytic Systems. Austin Chem Eng 5: 1060. Link: https://bit.ly/2yG3RO8

30. Michałowska-Kaczmarczyk AM, Michałowski T (2018) The modified Gran methods in potentiometric redox titrations derived according to GATES/GEB principles. Journal of Chemical Engineering and Bioanalytical Chemistry 2: 88 113. Link: https://bit.ly/3fwSFEb

31. Michałowska-Kaczmarczyk AM, Spórna-Kucab A, Michałowski T (2018) The balance $2 \cdot f(O)-f(H)$ as a keystone in formulation of electrolytic systems. Research and Reviews in Computational Chemistry 1: 1-9. Link: https://bit.ly/3bigSuk

32. Michałowska-Kaczmarczyk AM, Michałowski T (2018) The Balance 2·f(0) $f(H)$ as a Cornerstone in Formulation of Electrolytic Systems, Journal of New Developments in Chemistry 2: 1-13. Link: https://bit.ly/2WhrlCc

33. Michałowska-Kaczmarczyk AM, Michałowski T (2018) The distinguishing role of 2.f(O) $-f(H)$ in electrolytic systems. Biomed J Sci Tech Res 8: 1-10. Link: https://bit.ly/2WhOILP

34. Fuseler K, Krekeler D, Sydow U, Cypionka H (1996) A common pathway of sulfide oxidation by sulfate-reducing bacteria. FEMS Microbiol Lett 144: 129134. Link: https://bit.ly/2SRA9wB
35. Lange L, Triebel W (2000) Sulfides, Polysulfides, and Sulfanes. in Ullmann's Encyclopedia of Industrial Chemistry, Wiley-VCH, Weinheim. Link: https://bit.ly/3dz0xmR

36. Bo-Qing Xu, Sachtler WMH (1997) Reduction of SO4= ions in Sulfated Zirconia Catalysts. Journal of Catalysis 167: 224-233. Link: https://bit.ly/2WFcMqX

37. Meija J, Michałowska-Kaczmarczyk AM, Michałowski T (2017) Redox titration challenge. Anal Bioanal Chem 409: 11-13. Link: https://bit.ly/2SQUemB

38. Michałowski T, Michałowska-Kaczmarczyk AM, Meija J (2017) Solution of redox titration challenge. Anal Bioanal Chem 409: 4113-4115. Link: https://bit.ly/2yHkoBh

39. Michałowski T, Ponikvar-Svet M, Asuero AG, Kupiec K (2012) Thermodynamic and kinetic effects involved with $\mathrm{pH}$ titration of $\mathrm{As}$ (III) with iodine in a buffered malonate system. J Solution Chem 41: 436-446. Link: https://bit.ly/3boglYd

40. Standard Electrode Potentials. Link: https://bit.ly/3ciW5bE

41. Michałowska-Kaczmarczyk AM, Spórna-Kucab A, Michałowski T (2017) Dynamic Buffer Capacities in Redox Systems. Biochem Mol Biol J 1: 1-7. Link: https://bit.ly/3fCmSlo

42. Michałowska-Kaczmarczyk AM, Michałowski T (2015) Dynamic Buffe Capacity in Acid-Base Systems. J Solution Chem 44: 1256-1266. Link: https://bit.ly/3dmtMsW

43. Asuero AG, Michałowski T (2011) Comprehensive formulation of titration curves referred to complex acid-base systems and its analytical implications. Crit Rev Anal Chem 41: 151-187. Link: https://bit.ly/2WHXcL8

44. Michałowska-Kaczmarczyk AM, Michałowski T, Asuero AG (2015) Formulation of dynamic buffer capacity for phytic acid. Am J Chem Applications 2: 5-9. Link: https://bit.ly/3cdGv00

45. Michałowski T, Asuero AG (2012) New approaches in modelling the carbonate alkalinity and total alkalinity. Crit Rev Anal Chem 42: 220-244. Link: https://bit.ly/2SR4WcG

46. Michałowska-Kaczmarczyk AM, Michałowski T (2016) Application of Simms Constants in Modelling the Titrimetric Analyses of Fulvic Acids and Their Complexes with Metal Ions. J Solution Chem 45: 200-220. Link: https://bit.ly/2LejiQd

47. Karen P, McArdle P, Takats J (2014) Toward a comprehensive definition of oxidation state (IUPAC Technical Report). Pure and Applied Chemistry 86 1017-1081. Link: https://bit.ly/35luUV4

48. Karen P (2015) Oxidation state, a long-standing issue! Angewandte Chemie International Edition in English 54: 4716-4726. Link: https://bit.ly/3clnzgS

49. Karen P, McArdle P, Takats J (2016) Comprehensive definition of oxidation state (IUPAC Recommendations 2016). Pure and Applied Chemistry 88: 831839. Link: https://bit.ly/35J5I6p

50. Noether E (1918) Invariante Variationsproblem, Nachrichten der Königlichen Gesellschaft der Wissenschaften zu Göttingen Mathematisch-Physikalischen Klasse 1918: 235-237. Link: https://bit.ly/2WTKfOP

51. Michałowski T, Pilarski B, Dobkowska A, Młodzianowski J (2010) Mathematical modeling and physicochemical studies on acid-base equilibria in binary-solvent systems. Wiadomości Chemiczne 54: 124-154. Link: https://bit.ly/2T5dKMv

52. Pilarski B, Dobkowska A, Foks H, Michałowski T (2010) Modelling of acidbase equilibria in binary-solvent systems: A comparative study. Talanta 80 1073-1080. Link: https://bit.ly/3cjsaA2

53. Michałowski T, Pilarski B, Asuero AG, Michałowska-Kaczmarczyk AM (2014) Modeling of Acid-Base Properties in Binary-Solvent Systems. Handbook of Solvents 1: 23-648. Link: https://bit.ly/3cucuu1

Citation: AM Michałowska-Kaczmarczyk, Michałowski T (2020) Some detailed remarks on elemental and core balances formulated for non-redox and redox electrolytic systems. Biomed Sci Eng 6(1): 021-033. DOI: https://dx.doi.org/10.17352/abse.000017 
54. Michałowska-Kaczmarczyk AM, Michałowski T (2014) Generalized Electron Balance for Dynamic Redox Systems in Mixed-Solvent Media. Journal of Analytical Sciences, Methods and Instrumentation 4: 102-109. Link: https://bit.ly/3fsrASB
55. Michałowska-Kaczmarczyk AM, Asuero AG, Michałowski T (2015) "Why not stoichiometry" versus "Stoichiometry - why not?" Part I. General context. Crit Rev Anal Chem 45: 166-188. Link: https://bit.ly/3fxL3Bh
Discover a bigger Impact and Visibility of your article publication with Peertechz Publications

\section{Highlights}

* Signatory publisher of ORCID

- Signatory Publisher of DORA (San Francisco Declaration on Research Assessment)

* Articles archived in worlds' renowned service providers such as Portico, CNKI, AGRIS, TDNet, Base (Bielefeld University Library), CrossRef, Scilit, J-Gate etc.

* Journals indexed in ICMJE, SHERPA/ROMEO, Google Scholar etc.

* OAI-PMH (Open Archives Initiative Protocol for Metadata Harvesting)

* Dedicated Editorial Board for every journa

* Accurate and rapid peer-review process

* Increased citations of published articles through promotions

- Reduced timeline for article publication

Submit your articles and experience a new surge in publication services (https://www.peertechz.com/submission).

Peertechz journals wishes everlasting success in your every endeavours.

Copyright: @ 2020 AM Michałowska-Kaczmarczyk, et al. This is an open-access article distributed under the terms of the Creative Commons Attribution License, which permits unrestricted use, distribution, and r eproduction in any medium, provided the original author and source are credited.

Citation: AM Michałowska-Kaczmarczyk, Michałowski T (2020) Some detailed remarks on elemental and core balances formulated for non-redox and redox electrolytic systems. Biomed Sci Eng 6(1): 021-033. DOI: https://dx.doi.org/10.17352/abse.000017 